\title{
Receptores Multiusuários DS/CDMA Baseados na Projeção de Sinais em Subespaços Vetoriais
}

\author{
DS/CDMA Multiuser Detectors Based on Subspace \\ Methods
}

\author{
Jaime L. Jacob ${ }^{1}$ Taufik Abrão'; Paul Jean E. Jeszensky ${ }^{3}$
}

Resumo

\begin{abstract}
Neste trabalho são analisados detectores multiusuários (MuD) cegos e grupo-cegos (Bld-MuD e SBld-MuD, respectivamente) do ponto de vista do compromisso desempenho versus complexidade; especificamente, são caracterizados os detectores cegos e grupo-cegos baseados na inversão direta de matriz de correlação (DMI), no erro médio quadrático mínimo (MMSE) e os SBld-MuD híbridos nas formas I e II propostos em (H.-MADSEN; WANG, 2002). Simulações Monte Carlo (MCS) foram realizadas tendo em vista comprovar os resultados analíticos de desempenho, evidenciando-se a superioridade de desempenho do detector SBld-MuD. A contribuição deste trabalho consiste na análise comparada da degradação de desempenho dos três detectores, considerando erros nas estimativas dos parâmetros fase de portadora e coeficientes de canal Rayleigh (módulo e fase). Resultados MCS indicaram a superioridade de desempenho do detector SBld-MuD híbrido I na presença de erros nas estimativas dos três parâmetros.

Palavras-chave: DS/CDMA. Detecção Multiusuário. Detectores Grupo-cegos e Cegos. Vetores Ortogonais. Subespaço do Sinal.
\end{abstract}

\begin{abstract}
In this work blind and group-blind (Bld-MuD and SBld-MuD, respectively) multiuser detectors (MuD) are analyzed from the point of view of the trade-off between performance versus complexity; specifically, the blind and group-blind detectors are characterized based on the direct inversion of correlation matrix (DMI), the minimum mean square error (MMSE) and hybrid SBld-MuD in the forms I and II. Monte Carlo simulations (MCS) were carried out in order to prove analytical performance, being evidenced the superiority of SBld-MuD detectors. The contribution of this work consists of the compared analysis of the performance degradation for the three detectors, considering errors in the estimates of the channel and system parameters. MCS results indicated the superiority of the detector Hybrid SBld-MuD I, considering errors in the carrier phase and channel coefficients (module and phase) estimates.
\end{abstract}

Key words: Blind and Group-Blind Detection. Multiuser Detection. DS/CDMA, Orthogonal Vectors. Signal Subspace.

\footnotetext{
${ }^{1}$ Estudante de mestrado do Departamento de Engenharia Elétrica, Universidade Estadual de Londrina (DEEL-UEL), jljacob@uol.com.br

2 Professor Adjunto do DEEL-UEL; taufik@uel.br

${ }^{3}$ Professor Titular da Escola Politécnica da USP, PTC; pjj@1cs.poli.usp.br
} 


\section{Introdução}

Esse trabalho analisa técnicas avançadas de detecção da informação aplicáveis a sistemas de múltiplo acesso por divisão de código do tipo seqüência direta (DS/CDMA - Direct Sequence/Code Division Multiple Access). Tais sistemas utilizam a técnica de espalhamento espectral (SS - Spread Spectrum), em que o sinal transmitido ocupa uma banda muito maior que a banda da informação e cujo espalhamento é obtido por meio de um código independente da informação; particularmente, analisamse aqui técnicas de detecção grupo-cego em sistemas DS/CDMA. Nestes sistemas, a demodulação é acompanhada pela correlação do sinal recebido com uma réplica sincronizada do código de espalhamento usado na transmissão, assegurando a recuperação da informação no receptor.

Em sistemas DS/CDMA, o sucesso da detecção convencional depende das propriedades de correlação cruzada dos códigos de espalhamento envolvidos e de um rigoroso controle de potência. Vários métodos eficientes têm sido propostos considerando os mais variados cenários de comunicações, combinando diversos tipos de canal, transmissão e recepção. Na detecção multiusuário (ABRÃO, 2001), informações dos usuários ativos no sistema são utilizadas conjuntamente a fim de melhor detectar usuários de interesse, aumentando o desempenho e/ou a capacidade do sistema de comunicação móvel celular.

Em sistemas DS/CDMA, a limitação de desempenho é resultado principalmente da interferência de múltiplo acesso (MAI - Multiple Access Interference). Esta interferência é resultado dos offsets temporais aleatórios entre os sinais dos usuários ativos, tornando impossível o projeto de formas de onda de códigos de espalhamento completamente ortogonais. A MAI torna-se substancial quando o número de usuários cresce e/ou quando as potências dos usuários interferentes crescem. Nos enlaces reversos, a interferência é proveniente principalmente de outros transmissores móveis. No enlace direto, transmissões da estação rádio-base (ERB) de outras células (intercelulares), assim como os interferentes fortes da mesma base para outras estações móveis, podem resultar em fortes interferências para o sinal desejado. Em todos estes casos, tem-se o problema denominado "perto-longe"(near-far), em que os usuários interferentes localizados próximos ao receptor tendem a gerar muita interferência quando o sinal de interesse é transmitido a uma distância muito maior (longe), reduzindo tanto a qualidade de recepção e detecção quanto a capacidade do sistema como um todo.

Entre os MuD lineares, o descorrelacionador (SCHNEIDER, 1979), (LUPAS; VERDÚ, 1990) e o MMSE (VERDÚ, 1998) oferecem alta resistência ao efeito near-far. No entanto, são bastante sensíveis a erros nas estimativas de atrasos e amplitudes, particularmente o MMSE, e a carga de processamento para ambos ainda apresenta dificuldades de implementação utilizando processadores digitais de sinais (DSP - Digital Signal Processing), uma vez que requerem inversão de matriz.

Estimativas baseadas em subespaços usando técnicas como decomposição por valores singulares (SVD - Singular Value Decomposition) é uma ferramenta poderosa aplicável ao processamento de sinais em arranjo de antenas, análise de harmônicas, análise de fatores, identificação de sistemas, equalização cega de canais, estimativa de tempo, processamento de imagem, separação de sinal em comunicações de múltiplo acesso, entre outros. No caso da última aplicação, basicamente, a decomposição do sinal de múltiplo acesso DS/CDMA em subespaços vetoriais visa reduzir ou mesmo eliminar a interferência de múltiplo acesso (MAI) no processo de detecção multiusuário do sinal do usuário de interesse. Os MuD 
lineares baseados no princípio da projeção, também denominados de receptores baseados na projeção do sinais (PR - Projection Receiver) são muito atrativos por possuirem uma alta resistência ao efeito near-far (XU, 2000).

A análise do sinal na forma matricial produz autovalores e autovetores, os quais são empregados na obtenção de aproximações de baixo posto dos dados, visando reduzir a complexidade de implementação. Comumemente, são utilizadas as decomposições em valores singulares SVD ou em autovetores (EVD - Eigenvalue Decomposition) para a obtenção da análise do sinal sujeita à interferência e ruído. Assim, dado o vetor de sinais DS/CDMA recebido, $\mathbf{r}(t)$, a MAI pode ser eliminada projetando-se $\mathbf{r}(t)$ no subespaço ortogonal ao espaço vetorial constituído pelas sequiências de espalhamento de todos os sinais interferentes. Após esta projeção, teoricamente, obtém-se um modelo de sinais equivalente a um sistema DS/CDMA com um único usuário (single-user). O conjunto de bases ortogonais com espaço complementar ao espaço dos códigos interferentes é primeiro obtido pela decomposição SVD na correspondente linha/coluna da matriz de códigos, interferentes. Os dados recebidos amostrados são então projetados nesse espaço (técnica de detecção conjunta). Em seguida, aplica-se alguma técnica de detecção adequada para se obter estimativas confiáveis para os dados transmitidos.

Trabalhos recentes em detecção multiusuário mostram que os códigos usuários na ERB permite que a detecção de todos os usuários seja feita de forma simultânea. Se a detecção é feita na ERB de um sistema de comunicação móvel, os códigos de todos os usuários estão disponíveis. Por outro lado, se a detecção for feita na estação móvel, conhece-se somente o código do usuário de interesse. Portanto, nesse caso, pode-se utilizar o detector multiusuário cego (WANG; POOR, 1998), pois esses detectores ne- cessitam conhecer somente o código do usuário desejado.

Embora a ERB conheça todos os códigos dos usuários de dentro da célula, tipicamente não conhece os códigos dos usuários interferentes das células vizinhas. Isto é um sério problema para detectores multiusuários, já que em média um terço dos interferentes são oriundos de outras células, caracterizado a interferência intercelular (DUEL-HALLEN; ZVONAR, 1995). Os detectores cegos não usam o fato de que os códigos dos usuários intracelulares são conhecidos na ERB e também o fato de que esses outros usuários têm que ser detectados.

Isto leva a considerar detectores multiusuários que podem cancelar interferentes dos usuários conhecidos e desconhecidos utilizando as informações dos códigos de todos os usuários conhecidos e o fato de que a detecção pode ser feita simultaneamente para todos os usuários conhecidos. Quando muitos usuários são detectados conjuntamente, ter-se-á a vantagem da redução de complexidade computacional, se alguns dos processos forem comuns para todos os usuários. O detector que satisfaz a esses dois critérios denomina-se detectores multiusuários grupo-cegos (HOST-MADSEN, 1999), (HOST-MADSEN, 1998), (WANG; MADSEN, 1999) e (ZHANG; ZHANG, 2005).

As seções subsequentes estão organizadas da seguinte forma: a seção Modelo DE SISTEMA descreve o modelo vetorial de sistema DS/CDMA; a seção TÉCNICA de PROJEÇÃo EM SubespaÇoS apresenta a técnica de projeção em subespaço propriamente dita e a seção DESEMPENHO DOS DETECtores Multiusuário Grupo-Cegos e Cegos analisa desempenhos analíticos dos detectores lineares adaptativos grupo-cegos e cegos. Na seção RESULTADOS NUMÉRICOS são apresentados resultados de simulação Monte Carlo (MCS - Monte Carlo 
Simulation), tendo em vista confirmar a validade das equações analíticas de desempenho. Finalmente, as principais conclusões deste trabalho são apresentadas na seção CONCLUSÕES.

\section{Modelo de Sistema}

A seguir, apresenta-se o modelo matemático para o transmissor, canal e receptor DS/CDMA; o princípio de detecção Conv e alguns dos principais detectores $\mathrm{MuD}$ são discutidos; particularmente, mostrarse-ão as modificações necessárias em relação ao detector convencional para se obter MuDs baseados na projeção em subespaço.

\section{Detecção Convencional e MuD Lineares}

$\mathrm{O} i$-ésimo símbolo BPSK amostrado $\mathbf{x}(i)$ do sinal DS/CDMA transmitido pode ser escrito como, figura 1.(a):

$$
\mathbf{x}(i)=\sum_{k=1}^{K} \mathbf{h}_{k} b_{k}(i) \cos \left(\omega_{c} t+\varphi_{k}\right)
$$

onde $b_{k} \in\{ \pm 1\}$ é o conjunto de bits transmitidos, assumido valores equiprováveis e independentes; a matriz de bits é representada por $\mathbf{b}[i]=$ $\left[b_{1}[i] \cdots b_{K}[i]\right] ; \mathbf{h}_{k}(i)=F_{k}(i) \mathbf{s}_{k}$ é dado pelo produto do ganho do canal do $k$-ésimo usuário $F_{k}(i)=$ $A_{k}(i) G_{i}(k)$ e a seqüência de espalhamento normalizada; $K$ é o número total de usuários, $\omega_{c}=2 \pi f_{c}$ é a portadora e $\varphi_{k}$ a respectiva fase. A seqüência de espalhamento normalizada, empregada na transmissão do símbolo $b_{k}$, é definida por:

$$
\mathbf{s}_{k}(t)=\frac{1}{\sqrt{N}} \sum_{i=0}^{N-1} c_{k}[i] \psi\left(t-i T_{c}\right)
$$

onde $c_{k}[i] \in\{ \pm 1\}(0 \leq i \leq N-1)$ é o $i$-ésimo chip da sequiência de espalhamento alocada ao $k$ ésimo usuário e $\psi(t)$ é o pulso retangular de amplitude unitária com duração de $T_{c}=T / N$. A matriz de espalhamento de dimensão $N \times K$ é obtida através da concatenação dos vetores coluna $\mathbf{s}_{k}(t)$, onde $\mathbf{S} \triangleq\left[\mathbf{s}_{1} \cdots \mathbf{s}_{K}\right]$ é obtida amostrando-se (2) à taxa $\frac{1}{T_{c}}$.

Em canal com ruído branco Gaussiano aditivo complexo (AWGN - Additive White Gaussian Noise), representado por $n(t)$, tem-se valor médio do ruído igual a zero, densidade espectral de potência bilateral $\frac{N_{0}}{2}$ e potência de ruído $\sigma^{2} \cong N_{0} \cdot W=\frac{N_{0}}{T_{c}}$, onde $W$ é largura de banda ocupada pelo sistema de comunicação. Para o caso de sistema síncrono em canal AWGN, o coeficiente de canal, conforme figura 1.(b), $G_{k}(k=1, \ldots, K)$ resulta igual a 1, ou seja, não há desvanecimento de pequena escala no sinal recebido. Já em canal com desvanecimento, a amplitude do sinal recebido sofre profundas variações de acordo com o tempo e com distâncias compatíveis ao comprimento de onda da portadora; essa variação pode ser construtiva ou destrutiva; a esse fenômeno é dado o nome de desvanecimento de pequena escala do sinal. O coeficiente de canal para o sinal do $k$-ésimo usuário recebido, sujeito a desvanecimento Rayleigh plano, é dado por:

$$
G_{k}(t)=\alpha_{k}(t) e^{j \theta_{k}(t)}
$$

onde a envoltória $\alpha_{k}(t)$ apresenta distribuição estatística de Rayleigh e a fase $\theta_{k}(t)$, distribuição uniforme no intervalo $[0,2 \pi)$. Define-se ainda a matriz diagonal ganho de canal:

$$
\begin{aligned}
\mathbf{F}(t) & =\operatorname{diag}\left[F_{1}(t), F_{2}(t), \ldots F_{K}(t)\right] \\
& =\operatorname{diag}\left[A_{1} G_{1}(t), \ldots, A_{K} G_{K}(t)\right] \\
& =\operatorname{diag}\left[A_{1} \alpha_{1}(t) e^{j \theta_{1}(t)}, \ldots, A_{K} \alpha_{K}(t) e^{j \theta_{K}(t)}\right]
\end{aligned}
$$

onde $A_{k}$ é a amplitude do $k$-ésimo usuário, admitida constante ao longo de todo o quadro de bits transmitidos, constituindo a matriz quadrada, dimensão $K$ :

$$
\mathbf{A} \triangleq \operatorname{diag}\left(A_{1}, \ldots, A_{K}\right)=\left[\begin{array}{ccc}
A_{1} & & 0 \\
& \ddots & \\
0 & & A_{K}
\end{array}\right]
$$




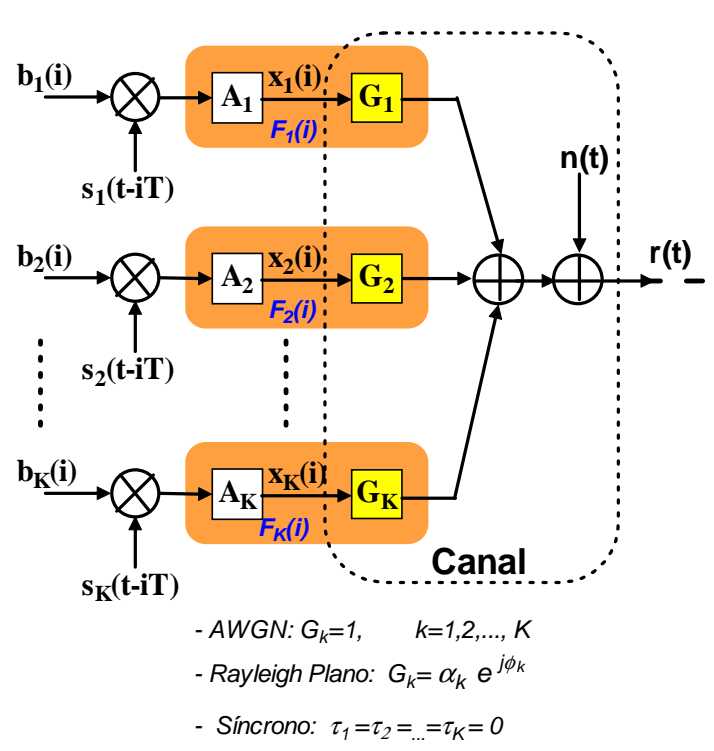

(a) Transmissor

(b) Canal

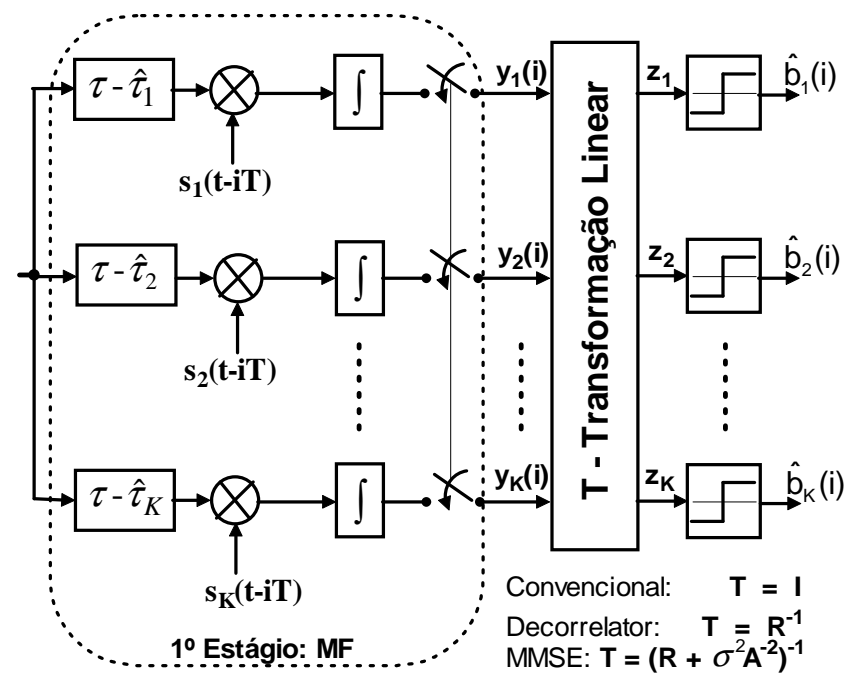

(c) Receptor

Figura 1: Modelo genérico em banda base equivalente discreto para um sistema DS/CDMA. a) transmissor equivalente; b) canal; c) receptor. $\mathbf{T}$ é a matriz de transformação linear.

Em sistemas DS/CDMA síncronos, tem-se $\tau_{k}=0$, para $k=1, \ldots, K$, figura 1.(c). Adicionalmente, considerando sistemas DS/CDMA intercelulares sujeitos ao desvanecimento Rayleigh plano, ter-se-á $\widetilde{K}$ usuários com códigos conhecidos e $\breve{K}$ usuários com códigos desconhecidos em um total de $K=$ $\widetilde{K}+\breve{K}$ usuários; nestas condições, o sinal recebido em banda base pode ser escrito como:

$$
r(t)=\sum_{k=1}^{\widetilde{K}} \widetilde{\mathbf{h}}_{k}(t) \widetilde{b}_{k}+\sum_{k=1}^{\breve{K}} \breve{\mathbf{h}}_{k}(t) \breve{b}_{k}+n(t),
$$

onde $t \in[0, T], \widetilde{\mathbf{h}}_{k}(t)=\widetilde{F}_{k}(t) \widetilde{\mathbf{s}}_{k}(t)$ é a matriz coluna do $k$-ésimo usuário conhecido, $\breve{\mathbf{h}}_{k}(t)=$ $\breve{F}_{k}(t) \breve{\mathbf{s}}_{k}(t)$ idem para $k$-ésimo usuário desconhecido (usuário intercelular), $\widetilde{b}_{k}$ e $\breve{b}_{k}$ são os respectivos bits transmitidos $( \pm 1)$, admitidos equiprováveis, e $n$ o AWGN. Definem-se ainda:

$$
\begin{aligned}
& \widetilde{\mathbf{S}} \triangleq\left[\mathbf{s}_{1} \cdots \mathbf{s}_{\widetilde{K}}\right], \\
& \breve{\mathbf{S}} \triangleq\left[\mathbf{s}_{\widetilde{K}+1} \cdots \mathbf{s}_{K}\right], \\
& \widetilde{\mathbf{F}} \triangleq \operatorname{diag}\left(F_{1}, \ldots, F_{\widetilde{K}}\right), \\
& \breve{\mathbf{F}} \triangleq \operatorname{diag}\left(F_{\widetilde{K}+1}, \ldots, F_{K}\right),
\end{aligned}
$$

$$
\begin{aligned}
\widetilde{\mathbf{b}}(t) & \triangleq\left[b_{1}(t) \cdots b_{\widetilde{K}}(t)\right], \\
\breve{\mathbf{b}}(t) & \triangleq\left[b_{\widetilde{K}+1}(t) \cdots b_{K}(t)\right], \\
\widetilde{\mathbf{H}}(t) & \triangleq\left[\mathbf{h}_{1}(t) \cdots \mathbf{h}_{\widetilde{K}}(t)\right], \\
\breve{\mathbf{H}}(t) & \triangleq\left[\mathbf{h}_{\widetilde{K}+1}(t) \cdots \mathbf{h}_{K}(t)\right] .
\end{aligned}
$$

Reescrevendo o sinal recebido (5) na forma vetorial, amostrado à taxa de $R_{c}=\frac{1}{T_{c}}$, tem-se:

$$
\mathbf{r}=\underbrace{\widetilde{\mathbf{H}} \widetilde{\mathbf{b}}}_{\text {conhecido }}+\underbrace{\breve{\mathbf{H}} \breve{\mathbf{b}}}_{\text {desconhecido }}+\mathbf{n}
$$

Reescrevendo (14) para o total de usuários $K$ tem-se (H.-MADSEN; WANG, 2002):

$$
\mathbf{r}[i]=\sum_{k=1}^{K} \mathbf{H}_{k} b_{k}+\mathbf{n}[i]=\mathbf{H b}+\mathbf{n}[i],
$$

onde $i=1,2, \ldots, M$, com $M=$ numero total de amostras processadas, $\mathbf{n}[i] \sim \mathcal{N}\left(0, \sigma^{2} \mathbf{I}_{N}\right)$ é o ruído Gaussiano branco aditivo de média zero e variância $\sigma^{2}$ e $\mathbf{I}_{N}$ é a matriz identidade $N \times N$.

O receptor em banda base da figura 1.(c) ao receber o sinal $\mathbf{r}$ faz a correlação com as sequiências 
de espalhamento dos respectivos usuários, passando então os sinais desespalhados por integradores e amostradores à taxa de bit de dados, $R=\frac{1}{T}$. A operação de desespalhamento é feita a partir de uma réplica da sequiência de espalhamento no receptor, sincronizada ao sinal recebido do usuário de interesse. Finalmente, estimativas de bits são obtidas comparando-se os sinais amostrados com o limiar zero em dispositivos de decisão de bits hard:

$$
\widehat{\mathbf{b}}=\operatorname{sgn}(\operatorname{Re}(\mathbf{z}))
$$

onde a métrica de decisão é dada por $\mathbf{z}=\mathbf{T y}$, com y a saída do banco de filtros casados; $\mathbf{T}$ é a matriz de transformação linear e $\operatorname{sgn}(\cdot)$ e $\operatorname{Re}(\cdot)$ são os operadores sinal de um número real e parte real de um número complexo, respectivamente. A decisão para o $k$-ésimo usuário é simplesmente:

$$
\begin{aligned}
\widehat{b}_{k}(i) & =\operatorname{sgn}\left\{\operatorname{Re}\left[z_{k}(i)\right]\right\} \\
& =\operatorname{sgn}\left\{\operatorname{Re}\left[\mathbf{t}_{k}^{H}(i) \mathbf{y}_{k}\right]\right\}= \begin{cases}-1, & z_{i}<0 \\
+1, & z_{i} \geq 0\end{cases}
\end{aligned}
$$

onde $\mathbf{t}_{k}$ é a $k$-ésima linha da matriz de $\mathbf{T}$.

$\mathrm{O}$ detector Convencional da figura 1.c obtém as decisões de bits realizando a transformação linear $\mathbf{T}=\mathbf{I}_{K}$ em $\mathbf{y}$, onde $\mathbf{I}_{K}$ é a matriz identidade de dimensão $K$, enquanto que para os receptores multiusuário linear de descorrelação esta transformação linear resulta em $\mathbf{T}=\mathbf{R}^{-1}$ e para o MMSE resulta $\mathbf{T}=\left(\mathbf{R}+\sigma^{2} \mathbf{A}^{-2}\right)^{-1}$, onde $\mathbf{R}$ é a matriz de correlação (auto e cruzada) normalizada definida por:

$$
\mathbf{R}=\mathbf{H}^{H} \mathbf{H}
$$

onde $\mathbf{H}$ é a matriz concatenada $\mathbf{H}=\left[\begin{array}{ll}\widetilde{\mathbf{H}} & \breve{\mathbf{H}}\end{array}\right]$. O descorrelacionador aplica a matriz de correlação inversa, $\mathbf{T}=\mathbf{R}^{-1}$, à saída do detector convencional objetivando o desacoplamento dos sinais dos usuários interferentes (VERDÚ, 1998), resultando em:

$$
\mathbf{z}(i)=\mathbf{R}^{-1} \mathbf{y}(i)=\mathbf{F}(i) \mathbf{b}(i)+\mathbf{v}(i)
$$

onde $\mathbf{v}(i)=\mathbf{R}^{-1} \mathbf{n}(i)$ é o vetor ruído filtrado (colorido).

O desempenho do descorrelacionador não é afetado pelas disparidades de potência dos usuários, possibilitando a detecção sem o conhecimento das amplitudes dos sinais recebidos. No entanto, a estimativa de dados para canais com desvanecimento depende do conhecimento das estimativas da fase dos coeficientes de canal:

$\widehat{b}_{k}(i)=\operatorname{sgn}\left\{\operatorname{Re}\left[z_{k}(i)\right]\right\}=\operatorname{sgn}\left\{\operatorname{Re}\left[z_{k}(i) e^{-j \theta_{k}(i)}\right]\right\}$

O detector multiusuário MMSE leva em conta o ruído de fundo, resultando em igual ou melhor desempenho, em termos de taxa de erro de bit (BER - bit error rate), que o detector descorrelacionador. Quando o ruído AWGN tender a zero, o detector MMSE converge, em termos de desempenho, para o descorrelacionador. No entanto, na obtenção do vetor de dados estimado é necessário conhecer as potências dos sinais recebidos. O detector MMSE substitui a transformação do descorrelacionador por:

$$
\mathbf{T}=\left(\mathbf{R}+\sigma^{2} \mathbf{A}^{-2}\right)^{-1}
$$

As saídas do MuD MMSE seguem as decisões:

$$
\widehat{b}_{k}(i)=\operatorname{sgn}\left(\operatorname{Re}\left(\left[\mathbf{R}+\sigma^{2} \mathbf{A}^{-2}\right]^{-1} \mathbf{y}\right)\right)_{k}
$$

Já o receptor com projeção em subespaço, figura 2, é constituído de um amostrador à taxa de chip amostrado, $R_{c}=1 / T_{c}$ e da formação, a partir do sinal recebido, de um vetor de projeção, que opera diretamente sobre o sinal recebido. O vetor projeção é obtido a partir das sequiências de espalhamento e por processos de decomposição de matrizes que será descrito nas seções seguintes. Na sequiência, será mostrado o vetor de recepção para o caso em que são considerados os usuários interferentes intracelulares, admitidos conhecidos e intercelulares, desconhecidos. 


\section{Estrutura de Detecção por Aproximação em} Subespaço

De forma análoga ao obtido com a estrutura de detecção descorrelacionadora e MMSE, o detector linear baseado na projeção em subespaço, para o $i$ ésimo usuário obtido aplicando-se a transformação linear $\mathbf{t}_{k}$ ao sinal recebido:

$$
\hat{b}_{i}=\operatorname{sgn}\left(\mathbf{w}_{i}^{H} \mathbf{r}\right)
$$

onde $\mathbf{w}_{i}$ é o vetor de projeção $N \times 1$. Para detectar simultaneamente todos os usuários, a equação (23) pode ser escrita como:

$$
\hat{\mathbf{b}}=\operatorname{sgn}\left(\mathbf{W}^{H} \mathbf{r}\right)
$$

onde $\mathbf{W}=\left[\begin{array}{llll}\mathbf{w}_{1} & \mathbf{w}_{2} & \ldots & \mathbf{w}_{K}\end{array}\right]$ é a matriz de projeção, dimensão $N \times K$. Este detector deve ter $\mathbf{w}_{i} \in$ $\operatorname{Im}\left(\left[\begin{array}{ll}\widetilde{\mathbf{H}} & \breve{H}\end{array}\right]\right)$, onde $\operatorname{Im}(\cdot)$ define espaço imagem ${ }^{4}$. Com isso, as componentes vetoriais de $\mathbf{w}_{i}$ que não pertencer a este subespaço contribuirão somente para o acréscimo de ruído, sem reduzir a interferência.

\section{Técnica de Projeção em Subespaços}

Projeção em subespaço é um método de separação de sinais na forma discreta a partir de um espaço vetorial de sinais. O detector MuD é especificamente projetado para rejeitar sinais interferentes, caracterizados pelas respectivas correlações cruzadas do sinal desejado, enquanto ainda é capaz de observar e detectar o sinal desejado.

\section{Auto-Decomposição de Sinais Decomposição em Valores Singulares}

Outra forma para se obter o subespaço desejado é o uso da decomposição em autovalores e autovetores

\footnotetext{
${ }^{4}$ Define-se imagem de uma matriz $\mathbf{A} \in \Re^{m \times n}$ como um subespaço em $\Re^{m}$ gerado pela imagem de $f(x)=\mathbf{A x}$ (MEYER, 2000): $\operatorname{Im}(\mathbf{A})=\left\{\mathbf{A x} \mid \mathbf{x} \in \Re^{n}\right\} \subseteq \Re^{m}$.
}

(EVD - eigendecomposition) ou decomposição em valores singulares (SVD - singular value decomposition) para detectores cegos (WANG; POOR, 1998). Por conveniência e sem perda de generalidade, assumese que as formas de onda de assinatura $\left\{\mathbf{s}_{k}\right\}_{k=1}^{K}$ dos $K$ usuários sejam linearmente independentes. A matriz de autocorrelação do sinal recebido é dada por:

$$
\mathbf{C}_{r}=\mathbb{E}\left\{\mathbf{r r}^{H}\right\}=\sum_{k=1}^{K} \mathbf{H}_{k} \mathbf{H}_{k}^{H}+\sigma^{2} \mathbf{I}_{N}=\mathbf{H H}^{H}+\sigma^{2} \mathbf{I}_{N} .
$$

onde $\mathbb{E}\{$.$\} é o operador esperança estatística. A$ autodecomposição da matriz $\mathbf{C}_{r}$ é dada por:

$$
\mathbf{C}_{r}=\mathbf{U} \Lambda \mathbf{U}^{H}=\left[\begin{array}{ll}
\mathbf{U}_{s} & \mathbf{U}_{n}
\end{array}\right]\left[\begin{array}{ll}
\Lambda_{s} & \\
& \Lambda_{n}
\end{array}\right]\left[\begin{array}{c}
\mathbf{U}_{s}^{H} \\
\mathbf{U}_{n}^{H}
\end{array}\right]
$$

onde $\mathbf{U}=\left[\mathbf{U}_{s} \mathbf{U}_{n}\right]$ é a matriz unitária de autovetores e $\Lambda=\operatorname{diag}\left(\Lambda_{s}, \Lambda_{n}\right)$ é a matriz de autovalores correspondentes; $\Lambda_{s}=\left(\lambda_{1}, \cdots, \lambda_{K}\right)$ contém os $K$ maiores autovalores de $\mathbf{C}_{r}$ em ordem descendente para detectores cegos; $\mathbf{U}_{s}=\left[\mathbf{u}_{1} \cdots \mathbf{u}_{K}\right]$ contém os correspondentes autovetores unitários ortogonais; $\Lambda_{n}=\sigma^{2} \mathbf{I}_{N-K}$ e $\mathbf{U}_{n}=\left[\mathbf{u}_{K+1} \cdots \mathbf{u}_{N}\right]$ contém os $(N-K)$ autovetores unitários ortogonais associados aos autovalores $\sigma^{2}$. O espaço abrangido por $\mathbf{U}_{s}$ é denominado de subespaço do sinal; enquanto que o espaço abrangido por $\mathbf{U}_{n}$ é denominado de subespaço do ruído.

\section{Projeção em Subespaços: MuD Grupo-Cego e Cego}

Em um sistema celular todos os códigos dos usuários e dentro de uma célula de interesse são conhecidos $(\widetilde{\mathbf{S}})$, enquanto, tipicamente, os códigos dos usuários interferentes fora da célula de interesse são desconhecidos $(\breve{\mathbf{S}})$. Estes usuários interferentes das células vizinhas é um sério problema para detecção multiusuário já que, em média, um terço dos interferentes são provenientes de outra célula (HOST-MADSEN, 


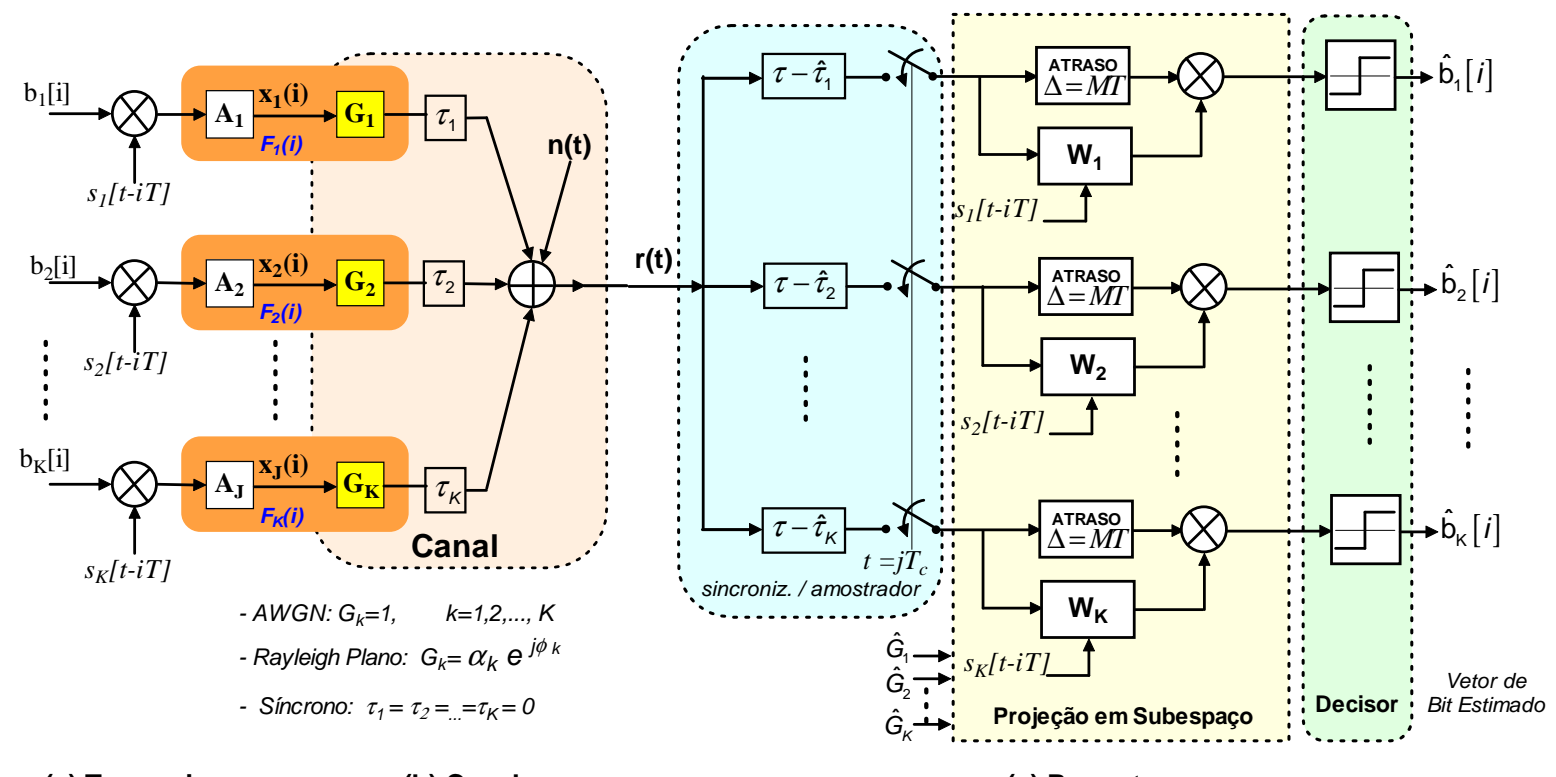

$\begin{array}{ll}\text { (a) Transmissor } & \text { (b) Canal }\end{array}$

(c) Receptor

Figura 2: Modelo genérico em banda base equivalente discreto para um sistema DS/CDMA. a) Transmissor eguivalente; b) Canal; c) Receptor. Detecção multiusuário com projeção em subespaço.

1998), ou seja, causam interferência de múltiplo acesso intercelular (VITERBI, 1994). Os detectores cegos (Bld-MuD), utilizando técnica de aproximação em subespaço (WANG; POOR, 1998) não consideram o fato dos códigos dos usuários interferentes intracelulares serem conhecidos pela ERB, somente o código do usuário de interesse é assumido conhecido.

Em (HOST-MADSEN, 1998) foi proposto um detector multiusuário a partir da utilização da técnica de aproximação em subespaço, que desacopla a interferência gerada pelos dois tipos de interferentes, os conhecidos (intracelulares) e desconhecidos (intercelulares); esta técnica utiliza as informações dos códigos dos usuários conhecidos e o fato de que a detecção é feita para todos os usuários conhecidos na ERB. Esse detector é denominado detector grupocego (SBld-MuD). Pelo fato de não envolver o conhecimento dos códigos dos usuários interferentes intercelulares, a detecção grupo-cega é claramente vantajosa, em termos do compromisso desempenho $\times$ complexidade computacional em relação à cega.
Baseado nos resultados de (H.-MADSEN; WANG, 2002), formula-se a seguir o problema da detecção MuD em canal AWGN síncrono, em que são conhecidas apenas as sequiências de espalhamento dos usuários pertencentes a uma determinada célula, enquanto os usuários das demais células são tratados como interferentes, porém desconhece-se seus parâmetros (número de usuários, sequiências de espalhamento, atrasos, ganhos de canal, etc). A este cenário denomina-se detecção $\mathrm{MuD}$ semi-cega ou grupo-cega.

\section{Desempenho dos Detectores Mul- tiusuário Grupo-Cegos e Cegos}

A dificuldade principal em se obter expressões confiáveis para o desempenho analítico dos métodos cegos está no fato de que as estimativas obtidas a partir dos sinais recebidos somente coincidirem com os sinais verdadeiros quando o número de sinais recebidos $M$ tende ao infinito. A partir do teorema de limite central, Madsen \& Wang (H.-MADSEN; WANG, 
2002) derivaram expressões analíticas para o desempenho de detectores cego e grupo-cego DS/CDMA em canais AWGN. Para canais AWGN síncronos, estas expressões resultam bem ajustadas aos resultados de simulação. A seguir, são apresentados os principais resultados de (H.-MADSEN; WANG, 2002) para canal AWGN síncrono.

\section{Desempenho Analítico de Detectores Linea- res Cegos e Grupo-Cegos}

Considere a expressão para o sinal DS/CDMA recebido (14). Um detector linear para o usuário 1, em (23), é um vetor determinístico $\mathbf{w}_{1} \in \mathcal{R}^{N}$ tal que $b_{1}[i]$ é demodulado de acordo com:

$$
\widehat{b}_{1}[i]=\operatorname{sgn}\left(\operatorname{Re}\left(\mathbf{w}_{1}^{T} \mathbf{r}[i]\right)\right)
$$

A saída deste $\mathrm{MuD}$ linear no tempo discreto $i$, correspondente ao período de bit $T$, é dada por:

$$
\mathbf{w}_{1}^{T} \mathbf{r}[i]=A_{1} b_{1}[i] \mathbf{w}_{1}^{T} \mathbf{s}_{1}+\sum_{k=2}^{K} A_{k} b_{k}[i] \mathbf{w}_{1}^{T} \mathbf{s}_{k}+\mathbf{w}_{1}^{T} \mathbf{n}[i]
$$

admitindo estimativa perfeita para a fase de portadora. O primeiro termo em (28) é o sinal projetado, o segundo termo é a MAI projetada no subespaço e o último termo é o ruído de fundo filtrado por $\mathbf{w}_{1}$. Assume-se que o fluxo de bit dos usuários e ruído são independentes entre si. A relação sinal-interferente mais ruído (SINR) na saída do detector linear $\mathbf{w}_{1}$ é dada por:

$$
\begin{aligned}
\operatorname{SINR}\left(\mathbf{w}_{1}\right) & \triangleq \frac{\mathbb{E}\left\{\mathbb{E}\left\{\mathbf{w}_{1}^{T} \mathbf{r}[i] \mid b_{1}[i]\right\}^{2}\right\}}{\mathbb{E}\left\{\operatorname{var}\left\{\mathbf{w}_{1}^{T} \mathbf{r}[i] \mid b_{1}[i]\right\}\right\}} \\
& =\frac{A_{1}^{2}\left(\mathbf{w}_{1}^{T} \mathbf{s}_{1}\right)^{2}}{\sum_{k=2}^{K} A_{k}^{2}\left(\mathbf{w}_{1}^{T} \mathbf{s}_{k}\right)^{2}+\sigma^{2}\left\|\mathbf{w}_{1}\right\|^{2}}
\end{aligned}
$$

onde $\mathbb{E}\left\{\mathbf{w}_{1}^{T} \mathbf{r}[i] \mid b_{1}[i]\right\}^{2}$ é o valor médio quadrático da projeção $\mathbf{w}_{1}^{T} \mathbf{r}[i]$, dado que $b_{1}[i]$ foi transmitido.

A BER do detector linear $\mathbf{w}_{1}$ é dada pela média aritmética das taxas de erros correspondentes a to- das as possíveis combinações dos bits dos usuários interferentes:

$$
\begin{aligned}
P_{e}\left(\mathbf{w}_{1}\right)= & P\left(\hat{b}_{1}[i] \neq b_{1}[i]\right) \\
= & \frac{1}{2^{K-1}} \sum_{\left[b_{2} \cdots b_{K}\right] \subset\{ \pm 1\}^{K-1}} \\
& Q\left(\frac{A_{1} \mathbf{w}_{1}^{T} \mathbf{s}_{1}+\sum_{k=2}^{K} A_{k} b_{k} \mathbf{w}_{1}^{T} \mathbf{s}_{k}}{\left\|\mathbf{w}_{1}\right\| \sigma}\right)
\end{aligned}
$$

onde a função $Q(\cdot)$ é definida a partir da função erro complementar, com $Q(x)=\frac{1}{2 \pi} \int_{x}^{\infty} e^{\frac{-u^{2}}{2}} d u=$ $\frac{1}{2} \operatorname{erf} c\left(\frac{x}{\sqrt{2}}\right)$.

A estimativa $\hat{\mathbf{w}}_{1}$ do $\mathrm{MuD} \mathbf{w}_{1}$ é obtida a partir do sinal recebido $\{\mathbf{r}[i]\}_{i=1}^{M}$, onde $M$ corresponde ao tamanho da janela de observação. Define-se $\Delta \mathbf{w}_{1} \triangleq$ $\hat{\mathbf{w}}_{1}-\mathbf{w}_{1}$. Aparentemente, $\hat{\mathbf{w}}_{1}$ e $\Delta \mathbf{w}_{1}$ são vetores aleatórios e são função das quantidades aleatórias $\{\mathbf{b}[i], \mathbf{n}[i]\}_{i=1}^{M}$. Em cenários típicos de detectores multiusuários adaptativos (WANG; POOR, 1998), a estimativa $\mathbf{w}_{1}$ é empregada em futuras demodulações do sinal recebido $\mathbf{r}[j], \quad j>M$. Assim, a saída do MuD linear é dada por:

$$
\hat{\mathbf{w}}_{1}^{T} \mathbf{r}[j]=\mathbf{w}_{1}^{T} \mathbf{r}[j]+\Delta \mathbf{w}_{1}^{T} \mathbf{r}[j], \quad j>M
$$

onde o primeiro termo em (31) representa a saída correta, que tem a mesma forma de (28). O segundo termo representa um termo de ruído adicional causado pelo erro na estimativa de $\mathbf{w}_{1}$. De (31), a SINR média de saída estimada é dado por (H.-MADSEN; WANG, 2002):

$\overline{\operatorname{SINR}\left(\hat{\mathbf{w}}_{1}\right)}=\frac{A_{1}^{2}\left(\mathbf{w}_{1}^{T} \mathbf{s}_{1}\right)^{2}}{\sum_{k=2}^{K} A_{k}^{2}\left(\mathbf{w}_{1}^{T} \mathbf{s}_{k}\right)^{2}+\sigma^{2}\left\|\mathbf{w}_{1}\right\|^{2}+X}$

$\operatorname{com} X=\mathbb{E}\left\{\left(\Delta \mathbf{w}_{1}^{T} \mathbf{r}[j]\right)^{2}\right\}=\frac{1}{M} \operatorname{tr}\left(\mathbf{C}_{w} \mathbf{C}_{r}\right)$ e as matrizes de correlação para o erro de estimativa para o sinal recebido são dadas, respectivamente por: $\frac{1}{M} \mathbf{C}_{w} \triangleq \mathbb{E}\left\{\Delta \mathbf{w}_{1} \Delta \mathbf{w}_{1}^{T}\right\}$ e $\mathbf{C}_{r} \triangleq \mathbb{E}\left\{\mathbf{r}[j] \mathbf{r}[j]^{T}\right\}$. O operador matricial $\operatorname{tr}(\cdot)$ é calculado pela soma dos 
elementos da diagonal principal de uma determinada matriz: $\operatorname{tr}(\mathbf{C})=\sum_{i=1}^{N} c_{i i}$ onde $\mathbf{C}$ tem dimensão $N$ $\times N$.

No processamento série dos bits de informação, o detector linear é usado para demodular o sinal $\mathbf{r}[i], 1 \leq i \leq M$. Desde que $\Delta \mathbf{w}_{1}$ é uma função de $\{\mathbf{r}[i]\}_{i=1}^{M}$, para valores fixos de $i, \Delta \mathbf{w}_{1}$ e $\mathbf{r}[i]$ são, em geral, correlacionados (H.-MADSEN; WANG, 2002). Entretanto, neste caso pode-se ainda empregar (32) como uma boa aproximação para SINR. Evidentemente, à medida que $M$ cresce, a correlação é reduzida e a estimativa para $\mathbf{w}_{1}$ melhorada.

A SINR média à saída do MuD estimado pode ser calculada a partir da matriz de autocorrelação $\mathbf{C}_{w}$. Pode-se mostrar através de simulação que a BER é bem aproximada por:

$$
\overline{P_{e}\left(\hat{\mathbf{w}}_{1}\right)} \cong Q\left(\sqrt{\overline{S I N R\left(\hat{\mathbf{w}}_{1}\right)}}\right)
$$

para os detectores cegos e grupo-cegos quando $M$ é razoavelmente grande.

Os detectores lineares MMSE cegos minimizam o valor do erro quadrático médio (MSE - mean-square error) entre a saída do detector e os bits de dados transmitidos dos usuários de interesse. $\mathrm{O}$ detector de inversão direta da matriz de correlação (DMI Direct-Matrix-Inversion) implementa diretamente a inversão da matriz de correlação $\mathbf{C}_{r}$, enquanto que detectores baseados em subespaço fazem a inversão após identificar um sinal-subespaço, projetando o sinal recebido neste subespaço. Ambos os detectores cegos MMSE e DMI podem ser implementados diretamente a partir do sinal recebido $\{\mathbf{r}[i]\}_{i=1}^{M}$, com conhecimento somente da forma de onda de espalhamento do usuário desejado $\mathbf{s}_{1}$. Para os detectores grupo-cegos, além de $\mathbf{s}_{1}$, a forma de onda de espalhamento de alguns outros usuários (usuários pertencentes à mesma célula do usuário de interesse) de- vem ser conhecidos no receptor. Assim, os detectores grupo-cegos suprimem os interferentes devido ao uso do código de espalhamento dos usuários conhecidos através do método de subespaço.

A partir do estabelecimento do teorema do limite assintótico, para cada uma das estimativas dos detectores acima, pode-se mostrar que, para valores fixos de $K$ e $N$, e conjuntos com valores fixos de sequiência de espalhamento, $\left\{\mathbf{s}_{k}\right\}_{k=1}^{K}$, o erro de estimativa para o detector MuD linear tende a uma distribuição Gaussiana (H.-MADSEN; WANG, 2002):

$$
\sqrt{M}\left(\hat{\mathbf{w}}_{1}-\mathbf{w}_{1}\right) \longrightarrow \mathcal{N}\left(\mathbf{0}, \mathbf{C}_{w}\right), \text { quando } M \rightarrow \infty
$$

A covariância do erro $\mathbf{C}_{w}$ é obtida para cada um dos detectores MuD lineares baseado em expressões aproximadas para $\overline{\text { SINR }}$ e BER dadas.

\section{A seção Resultados NumÉRicos mostra que} os detectores cegos baseados em subespaço superam o desempenho dos detectores cegos DMI em altas relações sinal-ruído SNR (signal-to-noise) e em regiões de baixa correlação cruzada dos sinais DS/CDMA; entretanto em baixa SNR e em regiões de alta correlação cruzada este resultado se inverte. Os detectores grupo-cego na forma II de (H.-MADSEN; WANG, 2002) são resistentes às condições de sistema onde os sinais resultam em altas correlações cruzadas, superando o desempenho dos detectores cegos. Já o detector grupo-cego na forma I de (H.-MADSEN; WANG, 2002) é resistente a ambas as condições de sistema: altas correlações cruzada e baixos SNR e portanto será analisado com mais cuidado neste trabalho.

\section{Limite Assintótico para os (S)Bld-MuD}

Nesta secção são apresentados os principais resultados do teorema do limite assintótico para os detectores multiusuários linear grupo-cego e cego. Esses re- 
sultados são baseados em (H.-MADSEN; WANG, 2002).

\section{Detector Cego MMSE Linear}

Considere novamente o sinal de múltiplo acesso recebido e amostrado em banda base, expresso por (15). O detector MMSE linear para o usuário 1 é definido como:

$$
\begin{aligned}
\mathbf{w}_{1}^{\text {mmse-i }} & =\min _{\mathbf{w} \in \mathcal{R}^{N}} \mathbb{E}\left\{\left(\frac{1}{\mu} b_{1}[i]-\mathbf{w}^{T} \mathbf{r}[i]\right)^{2}\right\} \\
& =\frac{A_{1}}{\mu} \mathbf{C}_{r}^{-1} \mathbf{s}_{1}
\end{aligned}
$$

com

$$
\mathbf{C}_{r} \triangleq \mathbb{E}\left\{\mathbf{r}[i] \mathbf{r}[i]^{T}\right\}=\mathbf{S S}^{T}+\sigma^{2} \mathbf{I}_{N}
$$

onde $\mu$ é uma constante positiva qualquer. Já que a regra de detecção linear (27) é invariante para um escalar positivo, o detector linear em (35) também é invariante para a constante positiva $\frac{A_{1}}{\mu}$. Para simplificar, escolhe-se $\mu=A_{1}$; portanto $\mathbf{w}_{1}^{\text {mmse-i }}=\mathbf{C}_{r}^{-1} \mathbf{s}_{1}$.

A autodecomposição da matriz de autocorrelação $\mathbf{C}_{r}$ em (36) é:

$$
\mathbf{C}_{r}=\mathbf{U}_{s} \Lambda_{s} \mathbf{U}_{s}^{T}+\sigma^{2} \mathbf{U}_{n} \mathbf{U}_{n}^{T}
$$

onde $\Lambda_{s}=\operatorname{diag}\left(\lambda_{1}, \ldots, \lambda_{K}\right)$ contém os $K$ maiores autovalores de $\mathbf{C}_{r} ; \mathbf{U}_{s}=\left[\mathbf{u}_{1}, \ldots, \mathbf{u}_{K}\right]$ contém os autovetores correspondentes aos $K$ maiores autovalores em $\Lambda_{s} ; \mathbf{U}_{n}=\left[\mathbf{u}_{K+1}, \ldots, \mathbf{u}_{N}\right]$ contém os $(N-K)$ autovetores correspondentes aos menores autovalores $\sigma^{2}$ de $\mathbf{C}_{r}$. Sabe-se que $\operatorname{Im}\left(\mathbf{U}_{s}\right)=\operatorname{Im}(\mathbf{S})$ é o subespaço do sinal e $\operatorname{Im}\left(\mathbf{U}_{n}\right) \perp \operatorname{Im}(\mathbf{S})$ é subespaço de ruído ${ }^{5}$. Assim, o detector MMSE linear $\mathbf{w}_{1} \mathrm{em}$ (35) também pode ser escrito em termos das componentes do subespaço do sinal (WANG; POOR, 1998):

$$
\mathbf{w}_{1}^{\text {mmse-ii }}=\mathbf{U}_{s} \Lambda_{s}^{-1} \mathbf{U}_{s}^{T} \mathbf{s}_{1} \quad \text { (Subespaços) }
$$

\footnotetext{
${ }^{5}$ Detalhes sobre imagem, ver (MEYER, 2000).
}

As eqs. (35) e (38) expressam a duas formas para o MuD linear MMSE cego, as quais assumem conhecimento da sequiência de espalhamento do usuário desejado $\mathbf{s}_{1}$ : método DMI e método de subespaços. No método DMI, a matriz de autocorrelação $\mathbf{C}_{r}$ em (35) é substituída pela correspondente amostra estimada:

$$
\begin{aligned}
\widehat{\mathbf{C}}_{r} & =\frac{1}{M} \sum_{i=1}^{M} \mathbf{r}[i] \mathbf{r}[i]^{T} \\
\widehat{\mathbf{w}}_{1} & =\widehat{\mathbf{C}}_{r}^{-1} \mathbf{s}_{1} \quad(\mathrm{DMI})
\end{aligned}
$$

Enquanto que no método de subespaços, os autocomponentes $\Lambda_{s}$ e $\mathbf{U}_{s}$ em (38) são substituídos pelos correspondentes autovalores e autovetores da matriz de autocorrelação estimada $\widehat{\mathbf{C}}_{r}$ :

$$
\begin{gathered}
\widehat{\mathbf{C}}_{r}=\frac{1}{M} \sum_{i=1}^{M} \mathbf{r}[i] \mathbf{r}[i]^{T}=\widehat{\mathbf{U}}_{s} \widehat{\Lambda}_{s} \widehat{\mathbf{U}}_{s}^{T}+\widehat{\mathbf{U}}_{n} \widehat{\Lambda}_{n} \widehat{\mathbf{U}}_{n}^{T} \\
\widehat{\mathbf{w}}_{1}=\widehat{\mathbf{U}}_{s} \widehat{\Lambda}_{s}^{-1} \widehat{\mathbf{U}}_{s}^{T} \mathbf{s}_{1} \quad \text { (Subespaço) }
\end{gathered}
$$

\section{MuD Lineares Grupo-Cego}

Assume-se aqui o conhecimento das formas de ondas das assinaturas dos usuários intracelulares $(\widetilde{K})$, mas não dos usuários intercelulares $(\breve{K})$. Em (15), admite-se que $\widetilde{\mathbf{S}}$ tenha posto de coluna completo. O detector híbrido grupo-cego linear reduz a zero a interferência causada pelos $\widetilde{K}$ usuários conhecidos e minimiza a interferência advinda dos restantes $\breve{K}$ usuários desconhecidos de acordo com o critério MMSE. Em particular, o detector grupo-cego para o usuário 1 é dado pela otimização do seguinte problema:

$$
\mathbf{w}_{1}=\arg \min _{\mathbf{w} \subset \mathcal{R}^{N}} \mathbb{E}\left\{\left(A_{1} b_{1}[i]-\mathbf{w}^{T} \mathbf{r}[i]\right)^{2}\right\}
$$

com $\mathbf{w}^{T} \widetilde{\mathbf{S}}=\tilde{\mathbf{e}}_{1}^{T}$, onde $\tilde{\mathbf{e}}_{k}$ é o $k$-ésimo vetor unitário em $\Re^{\tilde{K}}$. A solução de (43) é dada em termos das componentes do subespaço de sinal de $\mathbf{C}_{r}$ (WANG; 
MADSEN, 1999):

$$
\mathbf{w}_{1}=\mathbf{U}_{s} \Lambda_{s}^{-1} \mathbf{U}_{s}^{T} \widetilde{\mathbf{S}}\left(\widetilde{\mathbf{S}}^{T} \mathbf{U}_{s} \Lambda_{s}^{-1} \mathbf{U}_{s}^{T} \widetilde{\mathbf{S}}\right)^{-1} \tilde{\mathbf{e}}_{1}
$$

Assim, o detector SBld-MuD híbrido linear na forma II (SBld-II-MuD) é obtido substituindo-se as autocomponentes de $\mathbf{C}_{r}$ em (44) por aquelas da correspondente matriz de correlação estimada $\widehat{\mathbf{C}}_{r}$, equação (41).

Define-se a matriz projeção do sinal no subespaço ortogonal $\operatorname{ker}\left(\widetilde{\mathbf{S}}^{T}\right)$ como:

$$
\overline{\mathbf{P}} \triangleq \mathbf{I}_{N}-\widetilde{\mathbf{S}}\left(\widetilde{\mathbf{S}}^{T} \widetilde{\mathbf{S}}\right)^{-1} \widetilde{\mathbf{S}}^{T}
$$

onde o conjunto $\operatorname{ker}\left(\widetilde{\mathbf{S}}^{T}\right)=\left\{\mathbf{y}_{m \times 1} \mid \widetilde{\mathbf{S}}^{T} \mathbf{y}=\mathbf{0}\right\} \subseteq$ $\Re^{N}$, com $\widetilde{\mathbf{S}}$ de dimensão $N \times \widetilde{K}$, é denominado espaço nulo à esquerda de $\widetilde{\mathbf{S}}$, uma vez que $\operatorname{ker}\left(\widetilde{\mathbf{S}}^{T}\right)$ é o conjunto de todas as soluções do sistema homogêneo à esquerda $\mathbf{y}^{T} \widetilde{\mathbf{S}}=\mathbf{0}^{T}$. Pode-se facilmente mostrar que a matriz $\overline{\mathbf{P}} \mathbf{C}_{r} \overline{\mathbf{P}}$ apresenta autodecomposição na forma:

$\overline{\mathbf{P}} \mathbf{C}_{r} \overline{\mathbf{P}}=\left[\overline{\mathbf{U}}_{s} \overline{\mathbf{U}}_{n} \overline{\mathbf{U}}_{0}\right]\left[\begin{array}{ccc}\bar{\Lambda}_{s} & 0 & 0 \\ 0 & \sigma^{2} \mathbf{I}_{N-K} & 0 \\ 0 & 0 & 0\end{array}\right]\left[\begin{array}{c}\overline{\mathbf{U}}_{s}^{T} \\ \overline{\mathbf{U}}_{n}^{T} \\ \overline{\mathbf{U}}_{0}^{T}\end{array}\right]$

onde $\bar{\Lambda}_{s}=\operatorname{diag}\left(\bar{\lambda}_{1}, \ldots, \bar{\lambda}_{\breve{K}}\right)$, com $\bar{\lambda}_{k}>\sigma^{2}$; as colunas de $\overline{\mathbf{U}}_{s}$ formam uma base ortonormal para o subespaço $\operatorname{Im}(\mathbf{S}) \bigcap \operatorname{ker}\left(\widetilde{\mathbf{S}}^{T}\right)$ (MEYER, 2000). Finalmente, definindo-se $\widetilde{\mathbf{d}}_{1}$ e $\widetilde{\mathbf{m}}_{1}$ como, respectivamente, a transformação linear para o detector descorrelacionador e o detector MMSE para o usuário $1 \mathrm{e}$ assumindo-se que somente os primeiros $\widetilde{K}$ usuários estão presentes (WANG; MADSEN, 1999), tem-se:

$$
\begin{gathered}
\widetilde{\mathbf{d}}_{1} \triangleq \widetilde{\mathbf{S}}\left(\widetilde{\mathbf{S}}^{T} \widetilde{\mathbf{S}}\right)^{-1} \tilde{\mathbf{e}}_{1} \\
\widetilde{\mathbf{m}}_{1} \triangleq \widetilde{\mathbf{S}}\left(\widetilde{\mathbf{S}}^{T} \widetilde{\mathbf{S}}+\sigma^{2} \mathbf{I}_{\widetilde{K}}\right)^{-1} \tilde{\mathbf{e}}_{1}
\end{gathered}
$$

Desta forma, o detector híbrido grupo-cego linear, definido por (43), pode ser expresso em termos das componentes do subespaço de sinais de $\left(\overline{\mathbf{P}} \mathbf{C}_{r} \overline{\mathbf{P}}\right)$ como:

$$
\mathbf{w}_{1}=\left(\mathbf{I}_{N}-\overline{\mathbf{U}}_{s} \bar{\Lambda}_{s}^{-1} \overline{\mathbf{U}}_{s}^{T} \mathbf{C}_{r}\right) \tilde{\mathbf{d}}_{1}
$$

e analogamente, o detector MMSE grupo-cego linear é dado por:

$$
\mathbf{w}_{1}=\left(\mathbf{I}_{N}-\overline{\mathbf{U}}_{s} \bar{\Lambda}_{s}^{-1} \overline{\mathbf{U}}_{s}^{T} \mathbf{C}_{r}\right) \tilde{\mathbf{m}}_{1}
$$

Observe-se que o MuD grupo-cego da forma I (SBld-I-MuD) é formado pela substituição dos autocomponentes de $\overline{\mathbf{P}} \mathbf{C}_{r} \overline{\mathbf{P}}$ em (49) e (50) por aqueles das correspondentes amostragens estimadas $\left(\overline{\mathbf{P}} \widehat{\mathbf{C}}_{r} \overline{\mathbf{P}}\right)$ em (46).

\section{SINR de Saída para MuD Cegos}

Nesta subseção estabelece-se a SINR média de saída para detectores MMSE cegos.

SINR média. Considere $\mathbf{w}_{1}$ o detector MMSE linear verdadeiro dado por (35) [ou de forma equivalente, (38)], e $\widehat{\mathbf{w}}_{1}$ como sendo o detector MMSE cego linear estimado dado por (42). Considere (37) a autodecomposição da matriz de autocorrelação $\mathbf{C}_{r}$ do sinal recebido. Após longo desenvolvimento, obtém-se a SINR média de saída (H.-MADSEN; WANG, 2002):

$$
\overline{\operatorname{SINR}\left(\hat{\mathbf{w}}_{1}\right)}=\frac{A_{1}^{2}\left(\mathbf{w}_{1}^{T} \mathbf{s}_{1}\right)^{2}}{\sum_{k=2}^{K} A_{k}^{2}\left(\mathbf{w}_{1}^{T} \mathbf{s}_{k}\right)^{2}+\sigma^{2}\left\|\mathbf{w}_{1}\right\|^{2}+X}
$$

com

$$
\begin{aligned}
X= & \frac{1}{M}(K+1) \mathbf{w}_{1}^{T} \mathbf{s}_{1}+\frac{1}{M}(N-K) \xi \sigma^{2} \\
& -\frac{2}{M} \sum_{k=1}^{K} A_{k}^{4}\left(\mathbf{w}_{1}^{T} \mathbf{s}_{k}\right)^{2}\left(\mathbf{w}_{k}^{T} \mathbf{s}_{k}\right)
\end{aligned}
$$

onde:

$$
\mathbf{w}_{l}^{T} \mathbf{s}_{k}=\frac{1}{A_{l}^{2}}\left[\mathbf{R}\left(\mathbf{R}+\sigma^{2} \mathbf{A}^{-2}\right)^{-1}\right]_{k, l}, \quad k, l=1, \ldots, K
$$




$$
\begin{aligned}
\left\|\mathbf{w}_{1}\right\|^{2}= & \frac{1}{A_{1}^{4}}\left[\left(\mathbf{R}+\sigma^{2} \mathbf{A}^{-2}\right)^{-1} \mathbf{R}\left(\mathbf{R}+\sigma^{2} \mathbf{A}^{-2}\right)^{-1}\right]_{1,1} \\
\xi \sigma^{2} & =\mathbf{w}_{1}^{T} \mathbf{s}_{1} \quad(\mathrm{DMI}), \\
& =\frac{\sigma^{4}}{A_{1}^{4}}\left[\left(\mathbf{R}+\sigma^{2} \mathbf{A}^{-2}\right)^{-1} \mathbf{A}^{-2} \mathbf{R}^{-1}\right]_{1,1}
\end{aligned}
$$

(Subespaço)

e, analogamente a (18), $\mathbf{R}$ expressa a matriz de correlação considerando todos os usuários intra e intercelulares. Para sistemas síncronos, (18) é reduzida a $\mathbf{R}=\mathbf{S}^{T} \mathbf{S}$.

De (51), nota-se desempenho distintos para o detector cego DMI e o detector cego subespaço; esta diferença é causada pelo parâmetro $\xi$. Observese que o detector com menor $\xi$ resultará em maior SINR de saída. Considere $\mu_{1}, \ldots, \mu_{K}$ os autovalores da matriz $\mathbf{R}$. Define-se $\mu_{\min }=\min \left\{\mu_{k}\right\}$ e $\mu_{\max }=\max \left\{\mu_{k}\right\}, 1 \leq k \leq K$. Define-se também $A_{\min }=\min \left\{A_{k}\right\}$ e $A_{\max }=\max \left\{A_{k}\right\}$, $1 \leq k \leq K$. Assim,

se $\frac{A_{\min }^{2}}{\sigma^{2}}>\mu_{\max }, \Rightarrow \overline{S I N R}_{\text {subespaço }}>\overline{S I N R}_{\mathrm{DMI}}$

e $\overline{S I N R}_{\text {subespaço }}<\overline{S I N R}_{\text {DMI }}$, caso contrário. Esta é a condição para que o detector subespaço cego supere o DMI cego em termos da $\overline{\text { SINR }}$ de saída. Uma forma de se verificar a condição acima pode ser obtida em (H.-MADSEN; WANG, 2002).

\section{SINR de Saída para os MuD Grupo-Cegos}

Os resultados a seguir mostram a relação sinal-ruído mais interferência média para o detector híbrido grupo-cego forma II (SBld-II-MuD). As provas são encontradas em (H.-MADSEN; WANG, 2002).

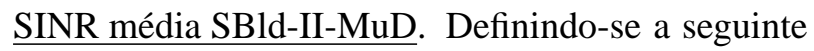
distribuição matricial:

$$
\mathbf{R}\left(\mathbf{R}+\sigma^{2} \mathbf{A}^{-2}\right)^{-1} \mathbf{A}^{-2}=\left[\begin{array}{cc}
\Psi_{11} & \Psi_{12} \\
\Psi_{21} & \Psi_{22}
\end{array}\right]
$$

onde $\Psi_{11}$ tem dimensão $\tilde{K} \times \tilde{K}$. Note-se que o lado direito da equação (57) é igual a $\left(\mathbf{S}^{T} \mathbf{U}_{s} \Lambda_{s}^{-1} \mathbf{U}_{s}^{T} \mathbf{S}\right)$ e portanto a matriz é simétrica. Definindo-se a matriz de dimensão $1: \tilde{K} \times 1: \tilde{K}$ :

$\Pi \triangleq\left[\mathbf{A}^{-2}\left(\mathbf{R}+\sigma^{2} \mathbf{A}^{-2}\right)^{-1} \mathbf{R}\left(\mathbf{R}+\sigma^{2} \mathbf{A}^{-2}\right)^{-1} \mathbf{A}^{-2}\right]$

a SINR de saída do detector híbrido grupo-cego linear forma II estimado é dada por:

$$
\overline{\operatorname{SINR}\left(\hat{\mathbf{w}}_{1}\right)}=\frac{A_{1}^{2}}{\sum_{k=1}^{\breve{K}} A_{\tilde{K}+k}^{2}\left(\mathbf{w}_{1}^{T} \mathbf{s}_{\tilde{K}+k}\right)^{2}+X}
$$

onde $X=\sigma^{2}\left\|\mathbf{w}_{1}\right\|^{2}+\operatorname{tr}\left(\mathbf{C}_{w} \mathbf{C}_{r}\right)$

$$
\begin{aligned}
& \mathbf{w}_{1}^{T} \mathbf{s}_{\tilde{K}+k}=\left[\Psi_{12}^{T} \Psi_{11}^{-1}\right]_{k, 1} \\
& \left\|\mathbf{w}_{1}\right\|^{2}=\left[\Psi_{11}^{-1} \Pi \Psi_{11}^{-1}\right]_{1,1}
\end{aligned}
$$

$$
\begin{array}{r}
\operatorname{tr}\left(\mathbf{C}_{w} \mathbf{C}_{r}\right)=\frac{1}{M}(N-K) \sigma^{4}\left[\Psi_{11}^{-1} \Pi \Psi_{11}^{-1}\right]_{1,1} \\
-2 \frac{1}{M} \sum_{k=1}^{\breve{K}} A_{\tilde{K}+k}^{4}\left[\Psi_{12}^{T} \Psi_{11}^{-1}\right]_{k, 1}^{2} \\
\cdot\left[\Psi_{22}-\Psi_{12}^{T} \Psi_{11}^{-1} \Psi_{12}\right]_{k, k}+\frac{1}{M}(\breve{K})\left[\Psi_{11}^{-1}\right]_{1,1}
\end{array}
$$

onde $\breve{\mathbf{S}} \triangleq\left[\mathbf{s}_{\tilde{K}+1}, \ldots, \mathbf{s}_{K}\right]$ e $\operatorname{tr}(\cdot)$ é o operador matricial traço.

SINR média SBld-I-MuD. Como antes, o SINR de saída para os SBld-I-MuD pode ser expresso em termos de $\mathbf{R}, \sigma^{2}$ e A. Entretanto, a expressão de SINR para este caso é trabalhosa e não será apresentada aqui devido a limitação de espaço.

\section{Resultados Numéricos}

Nesta secção são apresentados resultados numéricos através de simulações Monte Carlo visando confirmar a validade das equações analíticas de desempenho apresentadas na seção DESEMPENHO DOS DE- 
Tectores Multiusuário Grupo-Cegos e CEGOS. Todos os resultados foram obtidos utilizando ambiente MatLab versão 7.0. Considerou-se um sistema DS/CDMA síncrono, modulação BPSK, com código curto pseudo-Gold e ganho de processamento $N=15$; utilizou-se vários carregamentos, $L=$ $\frac{K}{N}=\frac{\widetilde{K}+\breve{K}}{N}$, mantido aproximadamente constante a razão entre usuários conhecidos e desconhecidos (interferentes intercelulares): $\frac{\widetilde{K}}{\widetilde{K}}=\frac{7}{4}$.

Foi utilizado um único conjunto de seqüência de Gold em que a cada nova iteração Monte Carlo houve a troca da seqüência da primeira linha (usuário de interesse) por uma outra, obtida de forma aleatória.

Em nossas simulações, utilizou-se o processo em série; neste procedimento, o detector linear estimado $\widehat{\mathbf{w}}_{1}$ é empregado na demodulação de todos os bits do usuário 1 a partir do vetor de recepção $\mathbf{r}[i]$. O comprimento do vetor recebido amostrado em banda base necessário para a estimativa a matriz de correlação é $i=1,2, \ldots, M$, enquanto que o comprimento dos quadros de bits transmitidos é dado por $j=$ $1,2, \ldots, F$ no qual necessariamente, $F>M$. Assim, o detector estimado $\widehat{\mathbf{w}}_{1}$ detecta todos os bits recebidos, para o usuário 1 inclusive aqueles utilizados para a formação da matriz de correlação.

A seguir são comparados os resultados de desempenho para os detectores MMSE linear cego por Inversão direta da matriz de correlação (DMI) (VERDÚ, 1998), (HONIG; VERDÚ, 1995) e o detector MMSE linear cego baseado em subespaço (WANG; POOR, 1998), o detector híbrido linear grupo-cego baseado em subespaço da forma I (WANG; MADSEN, 1999). A escolha do detector SblD-I-MuD se deve, conforme salientado anteriormente, à superioridade (ou igualdade) de desempenho em relação ao SblD-II-MuD.

Foram analisados resultados de desempenho para os casos AWGN e desvanecimento Rayleigh plano, com estimativas perfeitas e com erros. Assim, a degradação de desempenho dos Bld-MuD e SBldMuD foi analisada considerando erros nas estimativas de fase da portadora $\varphi_{k}$, de amplitude $\alpha_{k}$ e de fase $\theta_{k}$ dos coeficientes de canal. Estes erros foram introduzidos separada e/ou conjuntamente, sendo modelados através de distribuições estatísticas Gaussianas $\mathcal{N}$ :

$$
\begin{aligned}
\widehat{\varphi}_{k} & =\mathcal{N}\left[\frac{\epsilon_{\varphi}}{\pi}, \sigma_{\varphi}\right]+\varphi_{k} ; & & \text { (fase portadora) } \\
\widehat{\alpha}_{k} & =\mathcal{N}\left[g \epsilon_{\alpha}, \sigma_{\alpha}\right]+\alpha_{k} ; & & \text { (módulo coef. canal) } \\
\widehat{\theta}_{k} & =\mathcal{N}\left[\frac{\epsilon_{\theta}}{\pi}, \sigma_{\theta}\right]+\theta_{k} ; & & \text { (fase coef. canal) }
\end{aligned}
$$

$\operatorname{com} \epsilon_{\varphi}, \epsilon_{\alpha}$ e $\epsilon_{\theta} \in[0 ; 0,05 ; 0,10 ; 0,30]$, variância $\sigma^{2}=10^{-2}$ e a variável aleatória $g$ assumindo um dos dois valores $\{ \pm 1\}$ com igual probabilidade.

\section{Desempenho em Canais AWGN Síncronos}

As figuras 3 e 4 indicam, para $S N R=10 d B$ e controle perfeito de potência $(N F R=0 d B)$, resultados de desempenho, analíticos e de simulação Monte Carlo (MCS), para os dois primeiros detectores em função do número de bits $(M)$ usados para estimar a matriz de correlação. Para cada detector é plotado a BER, figura 3, e a SINR, figura 4.

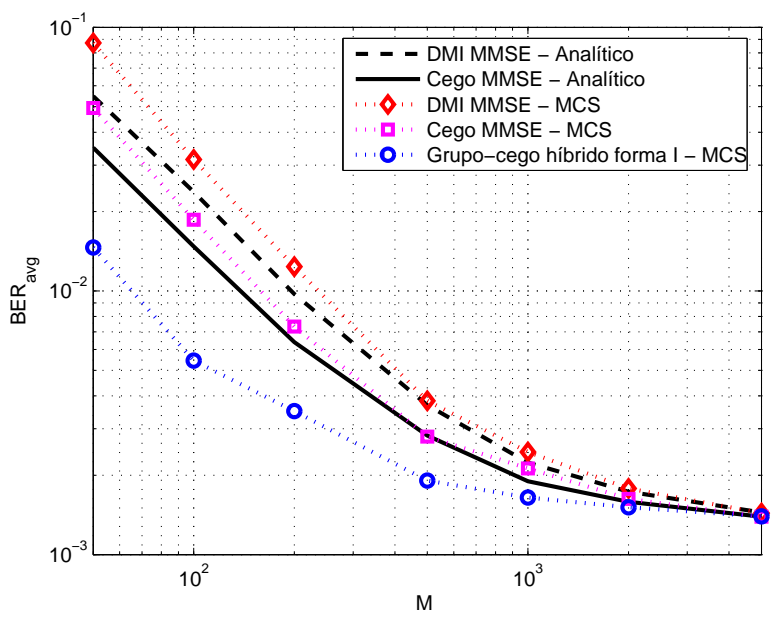

Figura 3: BER $\times$ número de amostras $M$ utilizadas na estimativa da matriz de correlação. $K=11, \widetilde{K}=$ 7, $S N R=10 d B, N F R=0 d B$. 
Observe-se que existe algum grau de discordância entre os desempenhos analíticos e os simulados para baixos valores de $M$. Isto é esperado, pois o desempenho analítico é baseado na análise assintótica. Pode ser visto que o SBld-I-MuD requer menos amostras $M$ para alcançar o mesmo desempenho dos demais detectores cegos (DMI e subespaço).

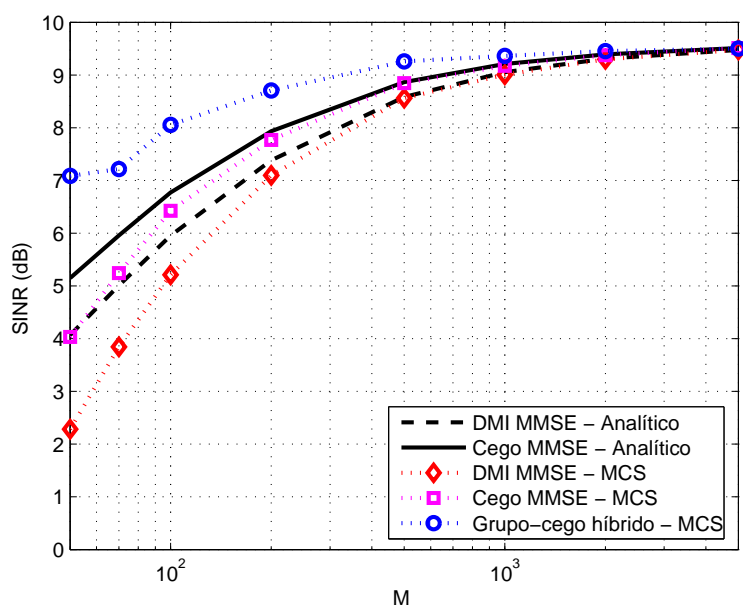

Figura 4: $\overline{S I N R} \times$ número de amostras $M$ do sinal e controle perfeito de potência, $K=11, \widetilde{K}=7$, $S N R=10 d B$.

Na figura 5 é apresentado resultado MCS para a SINR, em função do incremento no carregamento total do sistema. Para o cálculo do número de usuários conhecidos $\widetilde{K}$ foi mantida a proporcionalidade inicial entre usuários conhecidos em relação ao número de usuários total, $\frac{\widetilde{K}}{K} \approx$ cte $=\frac{7}{11} \mathrm{com}$ $K=3,4, \ldots, 15$ usuários. Para baixos valores de $K$, o ganho de desempenho do detector subespaço cego é maior e diminui à medida que $K$ aumenta. Pode ser visto também que o desempenho do detector DMI cego é insensível à variação do carregamento $K$. Pela incorporação da conhecida seqüência de espalhamento de outros usuários, o detector grupo cego oferece um melhor desempenho do que o detector DMI cego e o detector subespaço cego. Conforme é aumentado a quantidade de bits $M$, o desempenho do detector grupo-cego tende a se igualar ao do detector subespaço cego. Porém, quando $M$ é relativamente pequeno, o ganho de SINR do detector grupo-cego é maior em relação aos demais, evidenciando seu uso em aplicações nas quais não for possível operar com um grande número de bits $M$.

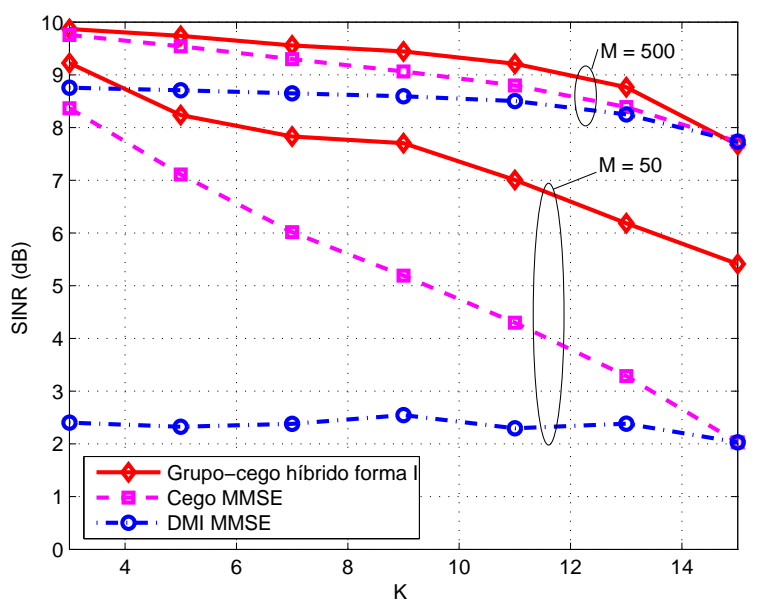

Figura 5: $\overline{S I N R}$ de saída versus o número total de usuários $K$ para os diferentes MuD lineares. Adotou-se $M=50$ e $500, S N R=10 d B$.

Para avaliar o desempenho dos MuD em relação ao efeito near-far, (NFR $\neq 0$ ), analisa-se na fi-

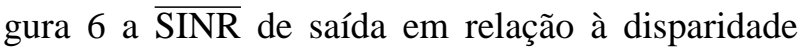
de potências dos usuários interferentes em relação ao usuário 1. Os usuários interferentes possuem as mesmas potências, ou seja, $A_{2}=\ldots=A_{K}$. O desempenho de todos os detectores é invariante para os diferentes valores de (NFR), ou seja, NFR = $20 \log \frac{A_{k}}{A_{1}}[d B]$ para $k=2, \ldots, K . \quad O$ detector grupo-cego supera o desempenho dos outros dois detectores, mas essa diferença é reduzida conforme o número de bits $M$ é incrementado, tendendo a se igualar quando $M \rightarrow \infty$. O detector grupo-cego apresenta uma deterioração na SINR de saída em torno de $2 d B$, quando $M=50$ em relação a $M \rightarrow$ $\infty$, enquanto que nos demais esta perda está em torno de $6,5 d B$ para o DMI e $5 d B$ para o MMSE.

A figura 7 analisa o efeito do aumento da interferência intercelular sobre o desempenho dos três $\mathrm{MuD}$, considerando distintas quantidades de bits de amostra $M$. Observe-se que para quantidade de 
amostras baixa, $M=50$, e $S N R=12 d B$, conforme a quantidade de usuários desconhecidos é incrementada o desempenho do detector grupo-cego decresce até atingir o desempenho dos outros dois detectores. Em contrapartida, para $M=500$ verifica-se que praticamente não há redução no desempenho do detector grupo-cego à medida que o número de usuários intercelulares cresce $\breve{K}$, até atingir o desempenho dos outros dois detectores.

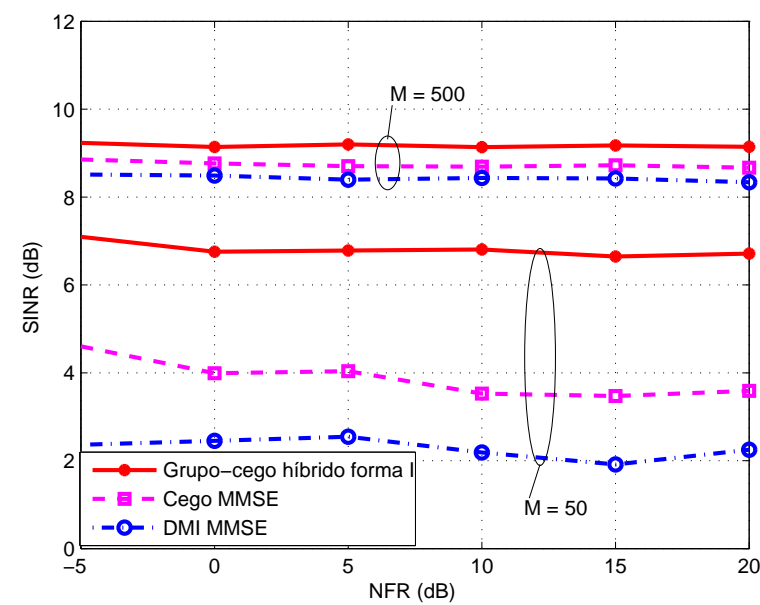

Figura 6: $\overline{S I N R}$ de saída para o usuário $1 \times$ desbalanço de potência dos usuários interferentes $A_{2}^{2}, \ldots, A_{K}^{2} \cdot K=11, \widetilde{K}=7, S N R=10 \mathrm{~dB}$ e quantidade de bits $M=50$ e 500 .

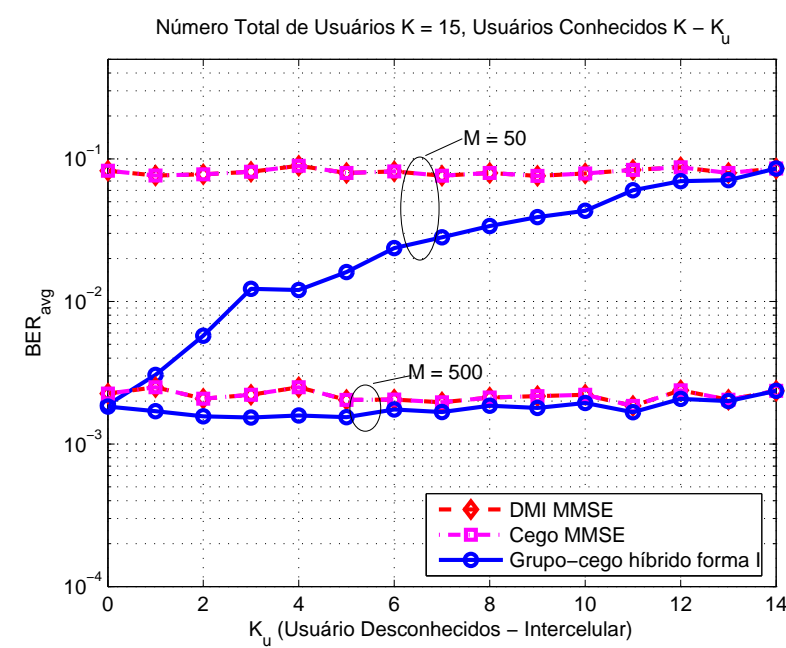

Figura 7: BER $\times$ quantidade de usuários desconhecidos $\breve{K}$ para os diferentes MuD lineares. $S N R=$ $12 d B, M=50$ ou $M=500, \widetilde{K}=K-\breve{K}=$ $15,14, \ldots, 1$.
A figura 8 confirma o melhor desempenho do detector Grupo-Cego em relação aos detectores DMI e os detectores cegos na análise da relação BER $\times$ SNR, principalmente nas regiões de médio e alto SNR. Essa figura evidencia a melhoria de desempenho dos detectores analisados com o aumento do número de bits $M$. Os desempenhos resultaram semelhantes nesses detectores para $S N R \leq 5 d B$ quando $M=200$ e para $S N R \leq 10 \mathrm{~dB}$, com $M=1000$, a partir do qual, o desempenho do SBldI-MuD supera significativamente o desempenho dos detectores DMI e o ceon

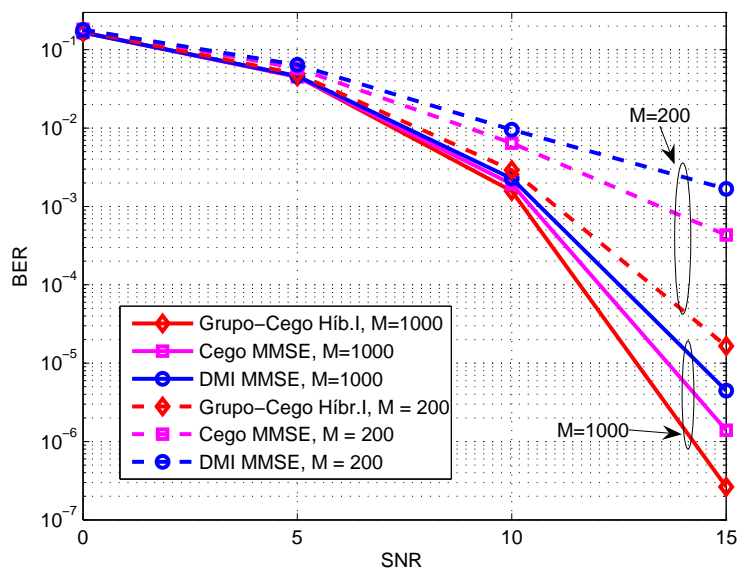

Figura 8: BER $\times$ SNR em canal AWGN com quadro de bits de valor fixo $F=60.000$, comprimento da amostra $M=200$ e $M=1000$.

\section{Desempenho em Canais AWGN com Erros nas Estimativas da Fase da Portadora}

Analisou-se a degradação de desempenho para os três detectores quando há erros nas estimativas da fase da portadora $\epsilon_{\varphi}$. Obteve-se a figura 9 considerando erros $\epsilon_{\varphi} \in[0,05 ; 0,30 ; 0,45]$ e $\sigma=0,1$, para comprimento da amostra $M=200$ e $M=$ 1000.

Analisando a degradação de desempenho para comprimento da amostra $M=1000$, figura 9.b, verifica-se a mesma tendência de degradação entre os detectores Grupo-Cego, Cego e DMI em relação 
ao comprimento da amostra $M=200$. No entanto, expressiva degradação de desempenho para os três detectores pôde ser observada quando o número de observações é reduzido de $M=1000$ para $M=200$, afetados por erros de até $\epsilon_{\varphi} \leq 0,10 \mathrm{com}$ variância fixada em $\sigma=10^{-2}$. Por sua vez, o detector DMI MMSE sofre a maior degradação em todas as condições analisadas $\left(M\right.$ e $\left.\epsilon_{\varphi}\right)$, atingindo o pior desempenho entre os três detectores analisados.

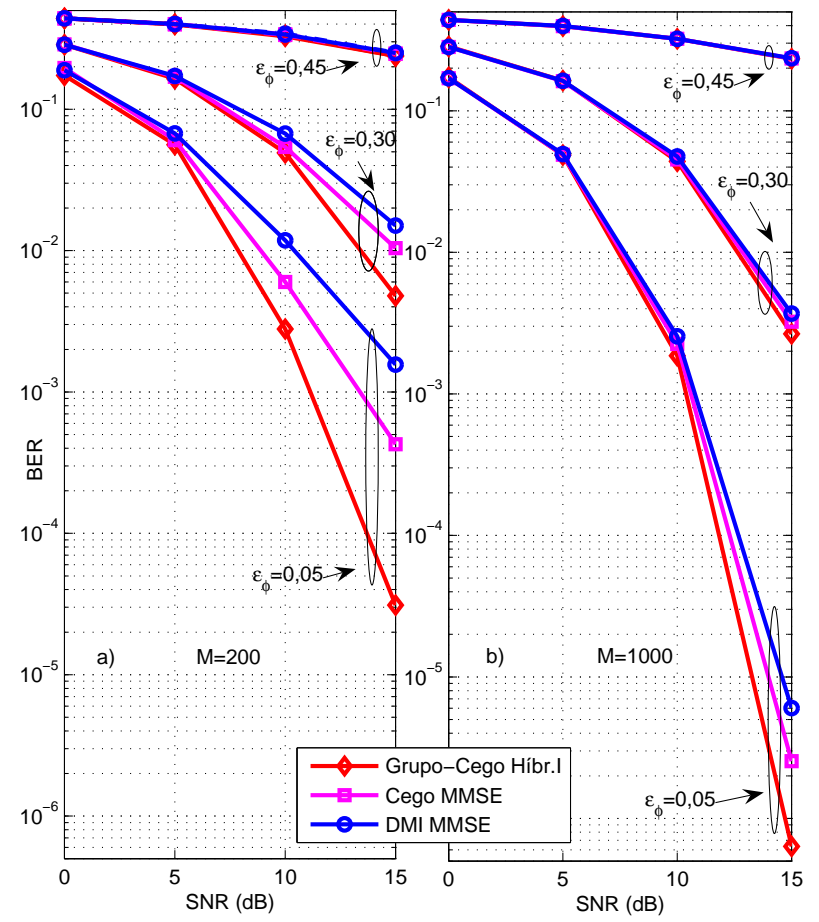

Figura 9: BER $\times$ SNR em canal AWGN com $F=$ 60.000 , erros na estimativa da fase da portadora $(\sigma=$ $0,1)$ e comprimento da amostra: a) $M=200$; b) $M=1000$.

Assim, sistemas DS/CDMA AWGN síncronos com detecção Grupo-cego, Cego e DMI são relativamente robustos aos erros nas estimativa da fase da portadora em até $10 \%$, porém essa degradacão será proporcionalmente ainda maior quando o comprimento das observações for pequena. A menor degradação relativa ocorre para o detector Grupocego.

\section{Desempenho em Canais com Desvanecimento}

Neste conjunto de resultados de simulação Monte Carlo foi considerado um sistema síncrono com desvanecimento Rayleigh plano e estimativas perfeitas para a fase da portadora e coeficientes de canal $\left(\epsilon_{\varphi}=\epsilon_{\alpha}=\epsilon_{\theta}=0\right)$.

Admitiu-se canal lentamente variável no tempo: os coeficientes de Rayleigh são constantes dentro de um intervalo de bit. Visando obter boas estimativas para a matriz de autocorrelação, adotou-se quadro de mesmo tamanho do comprimento da amostra, $M=F$. Analisou-se o desempenho do sistema no enlace direto, resultando o mesmo coeficiente de canal para todos os usuários simultaneamente. Adotouse unidades móveis com baixa velocidade (pedestre), resultando em valores de frequência Doppler máxima igual a $f_{D}=5 \mathrm{~Hz}$.

Adotando-se os mesmos parâmetros de sistema considerados anteriormente, obteve-se desempenhos para o sistema síncrono com desvanecimento plano caracterizado pelo piso de BER na região de alto SNR para o detector cego, figura 10.a. Incrementando-se o número de amostras para $M=$ 1000, figura 10.b, elimina-se o efeito de piso de BER, igualando-se o desempenho dos três sistemas na faixa de $E_{b} / N_{0}$ considerada.

\section{Sistemas em Canais com Desvanecimento e Erros nas Estimativas}

Resultados de desempenho com erros na estimativa do módulo dos coeficientes de canal $\epsilon_{\alpha}$ para os três sistemas são sintetizados na figura 11. Erros da ordem de até $\approx 30 \%$ nas estimativas do módulo dos coeficientes de canal para o sistema Grupo-Cego Híbrido síncrono em canal com desvanecimento plano $\left(\epsilon_{\alpha} \approx 0,30\right)$ não acarretaram degradação significativa de desempenho para os dois valores do 
número de amostras $(M=200$ e $M=1000)$, confirmando a robustez desta forma de detecção contra erros de estimativa no módulo do coeficiente de canal. Por sua vez, o detector Cego MMSE é sensível a erros nas estimativas do módulo dos coeficientes de canal quando o número de amostras é $<<1000$.

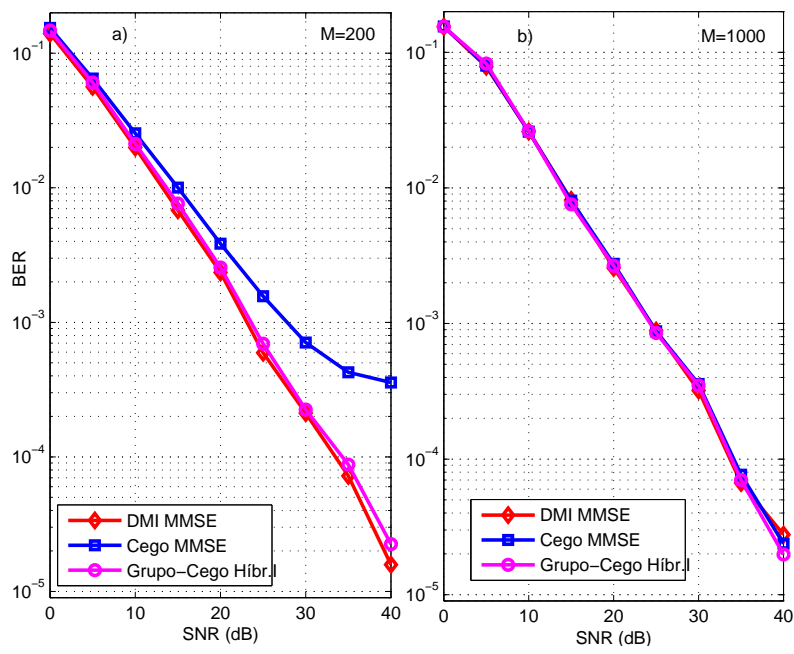

Figura 10: BER $\times$ SNR para o sistema síncrono em canal Rayleigh plano e estimativas perfeitas para coeficiente de canal: a) $F=M=200$; b) $F=M=$ 1000.

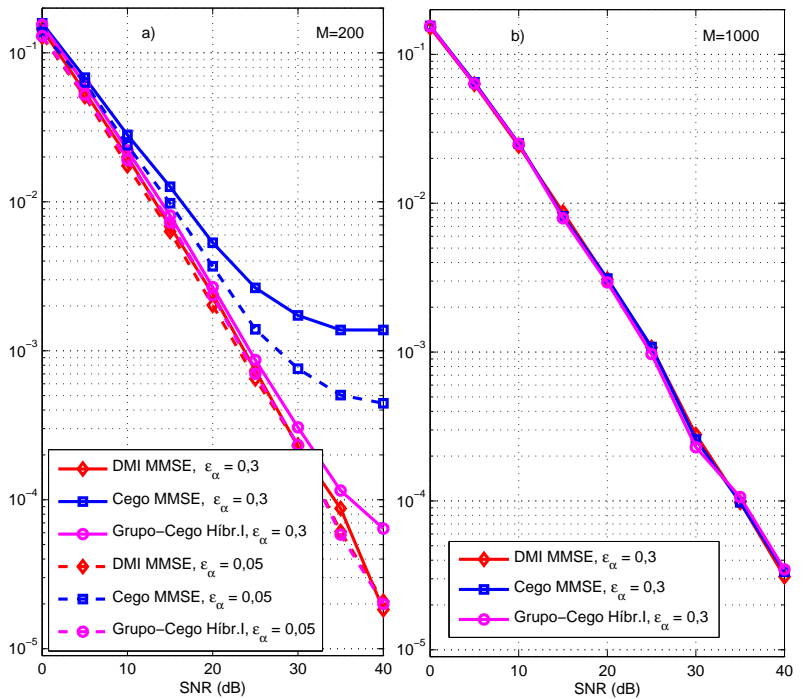

Figura 11: BER $\times$ SNR para o sistema síncrono em canal Rayleigh plano com erros nas estimativas do módulo dos coeficientes de canal. a) $F=M=200$; b) $F=M=1000$.

A degradação no desempenho dos três sistemas devido a erros nas estimativas das fases dos coeficiente de canal é mostrada na figura 12 . Foram considerados erros da ordem de $5 \%$ e $30 \%$ (i.e., $\epsilon_{\theta} \in$ $[0,05 ; 0,30])$ e o mesmo número de amostras do caso anterior. Observe-se que o detector Grupo-Cego Híbrido é mais sensível a erros na estimativas de fase do que no módulo dos coeficientes de canal (compare a degradação de desempenho na região de alto SNR nas figura 11 e 12), enquanto que a tendência contrária ocorre com o detector Cego MMSE, visível na condição $M=200$. Evidentemente, a robustez do detector DMI MMSE é obtida às custa de uma elevada complexidade devido à inversão da matriz de correlação, enquanto que o detector grupocego apresenta uma pequena degradação e uma menor complexidade de implementação.

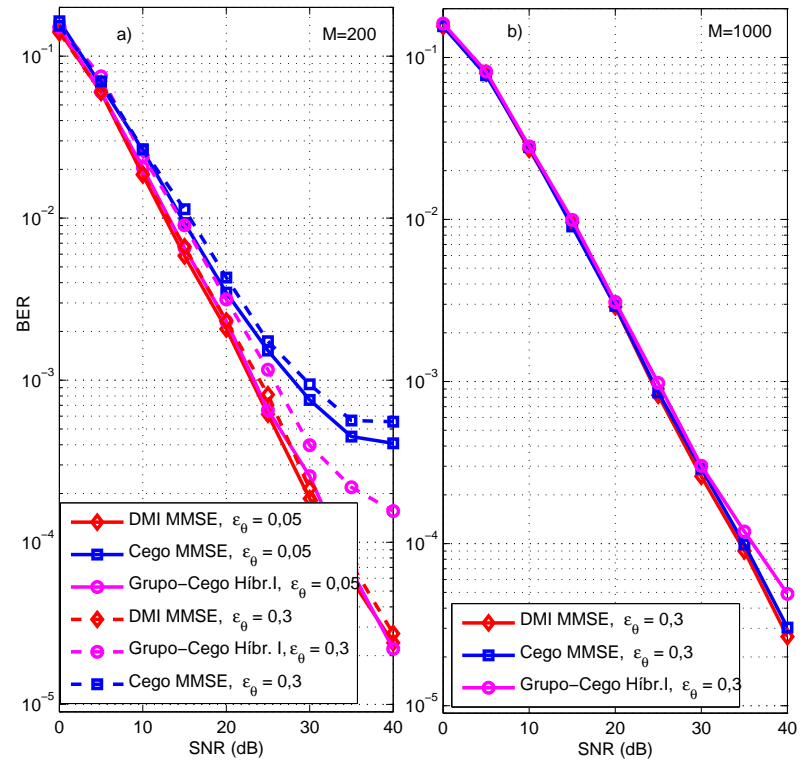

Figura 12: BER $\times$ SNR para o sistema síncrono em canal Rayleigh plano com erros nas estimativas da fase dos coeficientes de canal $(5 \%$ e $30 \%)$. a) $F=$ $M=200$; b) $F=M=1000$.

\section{Conclusões}

A utilização da técnica de projeção em subespaço na detecção multiusuário em sistemas DS/CDMA apresenta alguma degradação de desempenho para baixa 
relação sinal-ruído.

O detector descorrelacionador descrito na literatura tem desempenho inferior ou igual ao detector MMSE, dependendo da região de SNR, por isso a utilização do detector grupo-cego linear híbrido que combina as qualidades dos dois detectores citados é uma alternativa atraente.

O detector grupo-cego tem melhor desempenho do que os detectores cegos e os detectores não cegos, além de apresentar menor complexidade computacional. No entanto, requer o conhecimento dos parâmetros de sistemas de parte dos usuários interferentes (intracelulares).

Para os métodos em que é usado o vetor observação amostrado $\mathbf{r}[i]$, os resultados são imprecisos para um número pequeno de observações; porém o detector grupo-cego híbrido é capaz de operar satisfatoriamente, com pouca perda de desempenho e SINR quando o número de observações é relativamente pequeno $(M \approx 50)$.

Das três formas de detecção analisadas, o detector SBld-I-MuD apresentou a melhor robustez relativa ao erro de estimativa da fase da portadora tanto em sistemas síncronos com ruído AWGN (da ordem de $10 \%$ para o erro na estimativa de fase da portadora) quanto em sistemas síncronos com desvanecimento Rayleigh plano (da ordem de $30 \%$ para o erro de estimativa do módulo ou fase dos coefiicientes de canal). Particularmente, em canais com desvanecimento plano, algum tipo de diversidade deve ser incorporado aos detectores Cego e Grupo-Cego, quando o número de observações for pequeno, tendo em vista a redução ou mesmo eliminação do efeito do piso de BER.

\section{Referências}

ABRÃO, T. Canceladores de interferência multiusuário aplicados a sistemas DS/CDMA de múltipla taxa. Tese
(Tese de Doutorado) - Depto de Eng. Telecomunicações e Controle - EPUSP, Fevereiro 2001.

DUEL-HALLEN, J. H. A.; ZVONAR, Z. Multiuser detection for cdma systems. IEEE Pers. Commun., p. 46-58, April 1995.

H.-MADSEN, A.; WANG, X. Performance of blind and group-blind multiuser detectors. IEEE Trans. Information Theory, v. 48, n. 7, p. 1849-1872, 2002.

HONIG, U. M. M.; VERDÚ, S. Blind adaptive multiuser detection. IEEE Trans. Inform. Theory, v. 41, p. 994-960, July 1995.

HOST-MADSEN, A. Semi-blind decorrelating multiuser detectors for cdma: Subspace methods. In: IEEE PIMRC. Boston, USA: [s.n.], 1998.

HOST-MADSEN, A. Semi-Blind Multiuser Detectors for CDMA: Subspace Methods. University of Calgary, 1999.

LUPAS, R.; VERDÚ, S. Near-far resistence of multiuser detectors in asynchronous channels. IEEE Trans. Commun., v. 38, p. 496-508, April 1990.

MEYER, C. D. Matrix Analysis and Applied Linear Algebra. [S.1.]: SIAM, 2000.

SCHNEIDER, K. S. Optimum detection of code division multiplexed signals. IEEE Trans. Aerosp. Electron. Syst., v. 15, p. 181-185, January 1979.

VERDÚ, S. Multiuser Detection. Cambridge, UK: Cambridge Univ. Press, 1998.

VITERBI, A. J. The orthogonal-random wave form dichotomy for digital mobile comunications. IEEE Personal Communications, p. 18-24, First Quarter 1994.

WANG, X.; MADSEN, A. H. Group-blind multiuser detection for uplink cdma. IEEE J. Select. Areas Commun., v. 17, n. 11, p. 1971-1984, November 1999.

WANG, X.; POOR, H. V. Blind multiuser detection: A subspace aproach. IEEE Trans. Information Theory, v. 44, n. 2, p. 677-690, March 1998.

XU, Z. Asymptotically optimal cdma receiver: Perfect mai cancellation. In: IEEE (Ed.). Wireless Communications and Networking Conference, WCNC'00. [S.1.: s.n.], 2000. v. 3, p. $1496-1500$.

ZHANG, G. B. G.; ZHANG, L. Group-blind intersymbol multiuser detection for downlink cdma with multipath. IEEE J. Select. Areas Commun., v. 4, n. 2, p. 434-443, March 2005. 